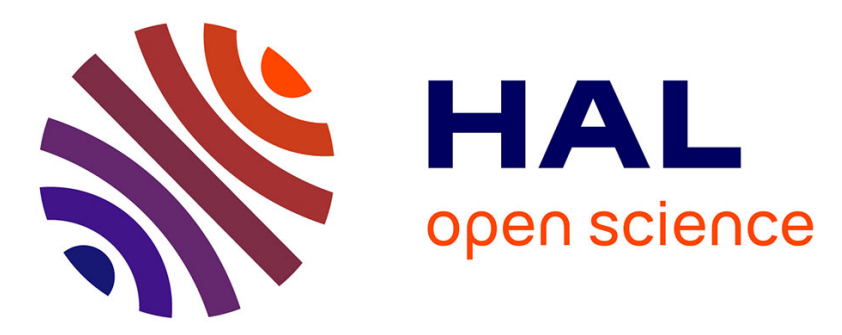

\title{
Stationary Oldroyd model with diffusive stress: mathematical analysis of the model and vanishing diffusion process
}

Laurent Chupin, Sébastien Martin

\section{- To cite this version:}

Laurent Chupin, Sébastien Martin. Stationary Oldroyd model with diffusive stress: mathematical analysis of the model and vanishing diffusion process. Journal of Non-Newtonian Fluid Mechanics, 2015, 218, pp.27-39. hal-00905096v2

\section{HAL Id: hal-00905096 \\ https://hal.science/hal-00905096v2}

Submitted on 8 Mar 2015

HAL is a multi-disciplinary open access archive for the deposit and dissemination of scientific research documents, whether they are published or not. The documents may come from teaching and research institutions in France or abroad, or from public or private research centers.
L'archive ouverte pluridisciplinaire HAL, est destinée au dépôt et à la diffusion de documents scientifiques de niveau recherche, publiés ou non, émanant des établissements d'enseignement et de recherche français ou étrangers, des laboratoires publics ou privés. 


\title{
Stationary Oldroyd model with diffusive stress: mathematical analysis of the model and vanishing diffusion process
}

\author{
Laurent Chupin* and Sébastien Martin ${ }^{\dagger}$
}

February 2, 2015

\begin{abstract}
In this paper we address the stationary Oldroyd model with diffusive stress in two ways: first we present the mathematical analysis of the model. Second, as the link between the diffusive model and the standard one is questionable from the mathematical point of view, we discuss, by means of numerical simulations, the behaviour of the model with respect to vanishing diffusion. In particular, numerical results in $2 \mathrm{D}$ or $3 \mathrm{D}$ suggest that the solution of the diffusive model converges to the solution of the non-diffusive model at order 1 in the $L^{2}$-norm and $H^{1}$-norm.
\end{abstract}

Keywords. viscoelastic fluid, Oldroyd model, diffusive stress

MSC. 76D03, 76A10, 35Q35

\section{Contents}

\section{Introduction}

*Université Blaise Pascal, Laboratoire de Mathématiques (CNRS UMR 6620), Campus des Cézeaux, 63177 Aubière cedex, France, laurent. chupin@math. univ-bpclermont.fr

†Université Paris Descartes, Laboratoire MAP5 (CNRS UMR 8145), 45 rue des Saints-Pères, 75270 Paris cedex 06, France, sebastien.martin@parisdescartes.fr 
2 Weak formulation of the stationary problem and main theoretical results 5

2.1 Proof of existence . . . . . . . . . . . . . . 7

2.2 Proof of uniqueness . . . . . . . . . . . . . . . . 11

2.3 Strong solutions . . . . . . . . . . . . . . . . 12

3 Numerical investigation of the vanishing diffusion process 13

3.1 Algorithm . . . . . . . . . . . . . . . . . . . 14

3.2 Flow past a cylinder in a plane channel in $2 \mathrm{D} \ldots \ldots \ldots$

3.2 .1 Numerical profiles . . . . . . . . . . . . . . . . 17

3.2 .2 Error analysis . . . . . . . . . . . . . . . . . . 17

3.3 Driven cavity in 2D . . . . . . . . . . . . . . . . . . . 19

3.3 .1 Numerical profiles . . . . . . . . . . . . . . . . . . . 19

3.3 .2 Error analysis . . . . . . . . . . . . . . . . . . . 19

3.4 Driven cavity in 3D . . . . . . . . . . . . . . . 20

3.4 .1 Numerical profiles . . . . . . . . . . . . . . . . . 20

3.4 .2 Error analysis . . . . . . . . . . . . . . . . 20

4 Concluding remarks 21

4.1 On the Johnson-Segalman model . . . . . . . . . . . . . 21

4.2 On the high Weissenberg regime . . . . . . . . . . . 23

\section{Introduction}

The Oldroyd model describes the behaviour of a viscoelastic fluid. Its principle is built upon a description of the shear stress that interpolates between a purely viscous contribution and a purely elastic contribution. Let $\Omega$ be a bounded open set in $\mathbb{R}^{3}$ and $\mathbf{f}$ be a given vector function on $\Omega$. We are looking for a vector function $\mathbf{u}: \Omega \mapsto \mathbb{R}^{3}$, a scalar function $p: \Omega \mapsto \mathbb{R}$ and a symmetric tensor function $\sigma: \Omega \mapsto \mathbb{R}^{3 \times 3}$, representing the velocity, the pressure and the elastic extra-stress of the fluid satisfying the following set of equations:

$$
\left\{\begin{aligned}
\operatorname{Re}(\mathbf{u} \cdot \nabla \mathbf{u})-(1-r) \Delta \mathbf{u}+\nabla p & =\operatorname{div} \boldsymbol{\sigma}+\mathbf{f}, & & \text { in } \Omega, \\
\operatorname{div} \mathbf{u} & =0, & & \text { in } \Omega, \\
\operatorname{We}\left(\mathbf{u} \cdot \nabla \boldsymbol{\sigma}+g_{a}(\nabla \mathbf{u}, \boldsymbol{\sigma})\right)+\boldsymbol{\sigma}-\mathrm{D} \Delta \boldsymbol{\sigma} & =2 r \mathbb{D}(\mathbf{u}), & & \text { in } \Omega, \\
\mathbf{u} & =\mathbf{0}, & & \text { on } \partial \Omega, \\
\mathrm{D} \partial_{\mathbf{n}} \boldsymbol{\sigma} & =\mathbf{0}, & & \text { on } \partial \Omega .
\end{aligned}\right.
$$


Here, Re denotes the Reynolds number that quantifies the inertial effects in the fluid flow; We is the Weissenberg number related to a relaxation time that characterizes the elasticity of the fluid; $\mathrm{D}$ is a diffusive parameter of the elastic stress; $r \in[0,1]$ is an interpolation parameter : cases $r \in(0,1)$ are often referred as Jeffreys models whereas case $r=1$ is referred as the Maxwell model. Besides, $\mathbb{D}(\mathbf{u})$ (resp. $\mathbb{W}(\mathbf{u})$ ) denotes the symmetric (resp. skew-symmetric) part of the velocity gradient. The function $g_{a},-1 \leq a \leq 1$, is a bilinear mapping related to the total derivative, in which the parameter $a$ interpolates between the so-called upper-convected model $(a=1)$ and lowerconvected model $(a=-1)$. Note that the case $a=0$ is known as the so-called corotational model. The function $g_{a}$ is defined as

$$
g_{a}(\nabla \mathbf{u}, \boldsymbol{\sigma})=\mathbb{W}(\mathbf{u}) \cdot \boldsymbol{\sigma}-\boldsymbol{\sigma} \cdot \mathbb{W}(\mathbf{u})+a(\mathbb{D}(\mathbf{u}) \cdot \boldsymbol{\sigma}+\boldsymbol{\sigma} \cdot \mathbb{D}(\mathbf{u})) .
$$

Remark 1 In standard derivations of Oldroyd model from kinetic models for dilute polymers, the diffusive term $\mathrm{D} \Delta \boldsymbol{\sigma}$ is routinely omitted, on the grounds that it is several orders of magnitude smaller than the other terms in the equation. It physically corresponds to a centre-of-mass diffusion term in the dumbell models and it is in the range of about $10^{-9}$ to $10^{-7}$ when the macroscopic length-scale of the domain is of order 1, see [3].

The Oldroyd model also has a transient version which, notably, has been extensively analysed in many ways: the transient version of the standard Oldroyd model (without diffusion: $D=0$ ) has been the subject of intensive studies: in particular P.-L. Lions \& N. Masmoudi [12] proved a global existence result of weak solutions for any data in the corotational case only. In the general case, C. Guillopé \& J.-C. Saut [10] proved the existence and uniqueness of local strong solutions; besides, if the fluid is not too elastic and if the data are sufficiently small, then solutions are global. Then L. Molinet \& R. Talhouk [13] proved that the smallness assumption on the elasticity of the fluid could be relaxed. The transient version of the diffusive Oldroyd model (with diffusion: $D>0$ ) has been studied by a few authors: recently P. Constantin \& M. Kliegl [7] proved the existence of global strong solutions in 2D for the Cauchy problem of the diffusive Oldroyd-B model (i.e. $a=+1$ ) and uniqueness of the solution among a class of strong solutions. Notice also that other regularizations of the standard Oldroyd model have been studied, see in particular [3].

Let us now discuss the stationary case. To our knowledge, the only available result is due to M. Renardy [16] and focuses on the standard model 
(without diffusion) only: existence and uniqueness of strong solutions is proved under the assumption of small regular data. The method used by M. Renardy is based on a reformulation of the Oldroyd model as a "Newtonian generalized" fluid: the contribution of the stress div $\boldsymbol{\sigma}$ is expressed as an implicit function of the velocity field $\mathbf{u}$ and, then, an iterative scheme is built upon this fully nonlinear system. When dealing with stress diffusion, the mathematical analysis of the model can be approached with a completely different framework, as the diffusive contribution in the stress equation drastically changes the mathematical properties of the system. So far, the method that we present in the present paper is based on a classical weak formulation and then on energy estimates. Let us underline the main differences with the standard Oldroyd model studied by M. Renardy: first, the diffusive model makes it possible to handle with irregular data and boundary conditions ; second, in the corotational case, the smallness of the data is not needed anymore.

Let us give some motivation for the study of the vanishing diffusion process which consists in investigating the asymptotic regime $\mathrm{D} \rightarrow 0$ in the diffusive Oldroyd model. As a matter of fact, the vanishing viscosity process describes the transition between a (second order) diffusive model towards a (first order) transport model. Thus, boundary conditions have to be discussed. For this, let us draw some parallel with the behaviour of scalar transport models, as the Oldroyd equation is a convection equation. In the framework of scalar conservation laws, a classical approach to deal with boundary conditions is to introduce the so-called vanishing viscosity method. But the analysis highly depends on the type of boundary conditions that are considered. In the case of Dirichlet boundary conditions, introducing artificial diffusion leads, when passing to the limit, to a problem in which boundary conditions cannot be assumed to be satisfied pointwise because of the degeneracy of the operator. Nevertheless, Bardos, LeRoux \& Nedelec [2] exhibited a condition that expresses the way the boundary conditions are satisfied at the limit. In particular, it includes the fact that when the flux is monotone, the boundary condition is active when information enters the domain whereas it is not active when it goes out. In the end, Bardos, LeRoux \& Nedelec provide a so-called weak entropy formulation, still based of the vanishing viscosity method, which leads to a well-posed problem: at the limit (i.e. without diffusion but with Dirichlet boundary conditions that have to be understood in a weak sense), the problem admits a unique solution. When dealing with Neumann boundary conditions, well-posedness in a similar set- 
ting is questionable: does the vanishing viscosity method may lead to the selection of a unique solution? To our knowledge, this is an open question. In the context of the diffusive Oldroyd model, as the order of mag nitude of D is about $10^{-7}$, the vanishing diffusion process has to be addressed. Besides, at least for the Oldroyd model, classical solutions do exist in the non-diffusive model [16] with suitable boundary conditions. Dealing with a system of conservation law (such as the Oldroyd model) is much more difficult than dealing with a scalar conservation law but numerical simulations may play a role in the analysis of the asymptotic regime $\mathrm{D} \rightarrow 0$ by providing ideas on the behaviour of the diffusive model with respect to the standard one.

The present paper is composed of three sections. In Section 2 we give the weak formulation of the problem, the mathematical framework and the main results: existence, uniqueness, regularity. Section 3 is devoted to the numerical simulation of the models: an algorithm is proposed and benchmark tests are considered in order to investigate the vanishing viscosity process.

\section{Weak formulation of the stationary prob- lem and main theoretical results}

For the sake of physical relevance, the mathematical analysis presented in this section is presented in dimension 3. However, results are valid in dimension 2. The variational formulation of Problem (1) is written

$$
\left\{\begin{array}{l}
\text { Find }(\mathbf{u}, \boldsymbol{\sigma}) \in V \times W \text { such that, for all }(\mathbf{v}, \boldsymbol{\tau}) \in V \times W \\
\operatorname{Re} \int_{\Omega}(\mathbf{u} \cdot \nabla \mathbf{u}) \cdot \mathbf{v}+(1-r) \int_{\Omega} \nabla \mathbf{u}: \nabla \mathbf{v}+\int_{\Omega} \boldsymbol{\sigma}: \mathbb{D}(\mathbf{v})=\langle\mathbf{f}, \mathbf{v}\rangle, \\
\operatorname{We} \int_{\Omega}\left(\mathbf{u} \cdot \nabla \boldsymbol{\sigma}+g_{a}(\nabla \mathbf{u}, \boldsymbol{\sigma})\right): \boldsymbol{\tau}+\int_{\Omega} \boldsymbol{\sigma}: \boldsymbol{\tau}+\mathrm{D} \int_{\Omega} \nabla \boldsymbol{\sigma}: \nabla \boldsymbol{\tau} \\
=2 r \int_{\Omega} \mathbb{D}(\mathbf{u}): \boldsymbol{\tau}
\end{array}\right.
$$

where the functional spaces $V$ and $W$ are defined by

$$
\begin{aligned}
& V=\left\{\mathbf{u} \in H_{0}^{1}(\Omega)^{3} ; \operatorname{div} \mathbf{u}=0\right\}, \\
& W=\left\{\boldsymbol{\sigma} \in H^{1}(\Omega)^{3 \times 3} ; \boldsymbol{\sigma}={ }^{T} \boldsymbol{\sigma}\right\},
\end{aligned}
$$


and where $\langle\cdot, \cdot\rangle$ denotes the duality bracket between $H^{-1}$ and $H_{0}^{1}$. We will use the following norms:

$$
\|\mathbf{u}\|_{V}^{2}=\int_{\Omega}\|\nabla \mathbf{u}\|^{2}, \quad\|\boldsymbol{\sigma}\|_{W}^{2}=\int_{\Omega}\|\boldsymbol{\sigma}\|^{2}+\|\nabla \boldsymbol{\sigma}\|^{2} .
$$

The main theorem which is proved in this article concerns an existence result for Problem (2). In its general form, it requires some assumptions on the data. For this, we introduce the following constants:

$$
\begin{aligned}
C_{(\mathrm{I})} & :=\frac{8|a| C_{\Omega}^{2} \mathrm{We}\|\mathbf{f}\|_{H^{-1}}}{\min (1-r, \mathrm{D})^{2}}, \\
C_{(\mathrm{II})} & :=\frac{\sqrt{2 r} \min (1-r, \mathrm{D})}{4|a| C_{\Omega}^{2} \mathrm{We}}\left(1-\sqrt{1-C_{(\mathrm{I})}}\right),
\end{aligned}
$$

where $C_{\Omega}$ is a constant which only depends on the domain $\Omega$.

\section{Remark 2}

1. Note that $C_{(\mathrm{II})}$ is defined provided $C_{(\mathrm{I})} \leq 1$.

2. For $a=0$, we have by continuity $C_{(\mathrm{I})}=0$ and $C_{(\mathrm{II})}=\frac{\sqrt{2 r}\|\mathbf{f}\|_{H^{-1}}}{\min (1-r, \mathrm{D})}$.

3. Constant $C_{\Omega}$ is related to the Sobolev injection $W \subset L^{4}(\Omega)^{3 \times 3}$ :

$$
\forall \boldsymbol{\sigma} \in W,\|\boldsymbol{\sigma}\|_{L^{4}} \leq C_{\Omega}\|\boldsymbol{\sigma}\|_{W}
$$

4. The only physical parameter that is not involved in the definition of constants $C_{(\mathrm{I})}$ and $C_{(\mathrm{II})}$ is the Reynolds number Re. In other words, the existence result that is further described does not depend on the value of the Reynolds number.

Theorem 1 Let $\Omega$ be a Lipschitz bounded open set in $\mathbb{R}^{3}$ and $\mathbf{f} \in H^{-1}(\Omega)^{3}$. Let $\mathrm{Re} \geq 0$, We $\geq 0,0<r<1,-1 \leq a \leq 1$ and $\mathrm{D}>0$.

- Existence. If $C_{(\mathrm{I})} \leq 1$ then, there exists a solution $(\mathbf{u}, \boldsymbol{\sigma})$ of problem (2) which satisfies

$$
2 r\|\mathbf{u}\|_{V}^{2}+\|\boldsymbol{\sigma}\|_{W}^{2} \leq C_{(\mathrm{II})}^{2} .
$$

Moreover there exists $p \in L^{2}(\Omega)$ such that $(\mathbf{u}, p)$ satisfies $(1)_{1,2,3}$ in the sense of distributions. 
- Uniqueness. Problem (2) admits at most one solution if one of the following conditions is satisfied:

a) $\|\mathbf{f}\|_{H^{-1}}$ is small enough;

b) Re and We are small enough.

- Regularity. If $\Omega$ is of class $\mathcal{C}^{\infty}$ and if each component of $\mathbf{f}$ belongs to $\mathcal{C}^{\infty}(\bar{\Omega})$ then each component of any solution of $(2)$ belongs to $\mathcal{C}^{\infty}(\bar{\Omega})$ and the considered solution satisfies (1) in a classical sense.

Let us mention two corollaries.

Corollary 2 Problem (2) admits a unique solution if one of the following conditions is satisfied:

a) $\|\mathbf{f}\|_{H^{-1}}$ is small enough;

b) Re and We are small enough.

Corollary 3 If $a=0$, there exists a solution for all data.

The proof is decomposed into four parts. In section 2.1 we show the existence for the weak formulation (2) using a Galerkin approximations and compactness results to perform the limit. The existence of a pressure is obtained by De Rham theory. In section 2.2, we prove the uniqueness of the solution and, in section 2.3, we investigate the regularity of the weak solutions and prove that, if the data are regular, so is the solution.

\subsection{Proof of existence}

As a preliminary, $V \times W$ is endowed with the scalar product $(\cdot, \cdot)_{V \times W}$ defined by

$$
((\mathbf{u}, \boldsymbol{\sigma}),(\mathbf{v}, \boldsymbol{\tau}))_{V \times W}=2 r(\mathbf{u}, \mathbf{v})_{V}+(\boldsymbol{\sigma}, \boldsymbol{\tau})_{W} .
$$

As $V$ and $W$ are separable Hilbert spaces, we consider a countable orthonormal basis $\left(\mathbf{w}_{k}\right)_{k \in \mathbb{N}}$ in the space $V$, and a countable orthonormal basis $\left(\mathbf{s}_{k}\right)_{k \in \mathbb{N}}$ in the space $W$. We use the notation $V_{k}:=\operatorname{span}\left(\mathbf{w}_{1}, \ldots, \mathbf{w}_{k}\right)$ and $W_{k}:=\operatorname{span}\left(\mathbf{s}_{1}, \ldots, \mathbf{s}_{k}\right)$. For each fixed integer $k \in \mathbb{N}$, we would like to define an approximate solution $\left(\mathbf{u}_{k}, \boldsymbol{\sigma}_{k}\right)$ of (2) by

$$
\mathbf{u}_{k}=\sum_{i=0}^{k} \alpha_{i, k} \mathbf{w}_{i}, \quad \boldsymbol{\sigma}_{k}=\sum_{i=0}^{k} \beta_{i, k} \mathbf{s}_{i},
$$


satisfying the variational problem

$$
\begin{array}{r}
\operatorname{Re} \int_{\Omega}\left(\mathbf{u}_{k} \cdot \nabla \mathbf{u}_{k}\right) \cdot \mathbf{v}_{k}+(1-r) \int_{\Omega} \nabla \mathbf{u}_{k}: \nabla \mathbf{v}_{k}+\int_{\Omega} \boldsymbol{\sigma}_{k}: \mathbb{D}\left(\mathbf{v}_{k}\right)=\left\langle\mathbf{f}, \mathbf{v}_{k}\right\rangle, \\
\operatorname{We} \int_{\Omega}\left(\mathbf{u}_{k} \cdot \nabla \boldsymbol{\sigma}_{k}+g_{a}\left(\nabla \mathbf{u}_{k}, \boldsymbol{\sigma}_{k}\right)\right): \boldsymbol{\tau}_{k}+\int_{\Omega} \boldsymbol{\sigma}_{k}: \boldsymbol{\tau}_{k}+\mathrm{D} \int_{\Omega} \nabla \boldsymbol{\sigma}_{k}: \nabla \boldsymbol{\tau}_{k} \\
=2 r \int_{\Omega} \boldsymbol{\tau}_{k}: \mathbb{D}\left(\mathbf{u}_{k}\right),
\end{array}
$$

for all $\left(\mathbf{v}_{k}, \boldsymbol{\tau}_{k}\right) \in V_{k} \times W_{k}$. Equations (4) form a system of nonlinear equations for $\alpha_{1, k}, \ldots, \alpha_{k, k}, \beta_{1, k}, \ldots, \beta_{k, k}$, and the existence of a solution of this system is not obvious. We use the following lemma:

Lemma 1 Let $X$ be a finite dimensional Hilbert space with scalar product $(\cdot, \cdot)$ and norm $\|\cdot\|$, and let $P$ be a continuous mapping from $X$ into itself such that

$$
\exists R>0 ; \forall \xi \in X \quad(\|\xi\|=R \Longrightarrow(P(\xi), \xi) \geq 0) .
$$

Then there exists $\xi \in X,\|\xi\| \leq R$, such that $P(\xi)=0$.

The related proof, based on the Brouwer fixed point theorem, can be found in $[17$, p.166]. We only note that the result which is proved in [17] corresponds to the case where the inegality $(P(\xi), \xi) \geq 0$ in the assertion (5) is a strict inequality. The case of a large inequality holds too, with the same proof.

We apply this lemma to prove the existence of $\left(\mathbf{u}_{k}, \boldsymbol{\sigma}_{k}\right)$ as follows: let $X$ be the space defined as $X:=V_{k} \times W_{k}$, endowed with the scalar product inherited from $V \times W$. Let $P_{k}$ the mapping from $X$ into itself defined by, for all $((\mathbf{u}, \boldsymbol{\sigma}),(\mathbf{v}, \boldsymbol{\tau})) \in X^{2}$

$$
\begin{aligned}
&\left(P_{k}(\mathbf{u}, \boldsymbol{\sigma}),(\mathbf{v}, \boldsymbol{\tau})\right)_{X} \\
&=2 r {\left[\operatorname{Re} \int_{\Omega}(\mathbf{u} \cdot \nabla \mathbf{u}) \cdot \mathbf{v}+(1-r) \int_{\Omega} \nabla \mathbf{u}: \nabla \mathbf{v}+\int_{\Omega} \boldsymbol{\sigma}: \mathbb{D}(\mathbf{v})-\langle\mathbf{f}, \mathbf{v}\rangle\right] } \\
&+ {\left[\int_{\Omega} \boldsymbol{\sigma}: \boldsymbol{\tau}+\mathrm{D} \int_{\Omega} \nabla \boldsymbol{\sigma}: \nabla \boldsymbol{\tau}+\mathrm{We} \int_{\Omega}\left(\mathbf{u} \cdot \nabla \boldsymbol{\sigma}+g_{a}(\nabla \mathbf{u}, \boldsymbol{\sigma})\right): \boldsymbol{\tau}\right.} \\
&\left.-2 r \int_{\Omega} \mathbb{D}(\mathbf{u}): \boldsymbol{\tau}\right] .
\end{aligned}
$$


The continuity of $P_{k}$ is obvious. Let us show that condition (5) holds. Denoting $\xi=(\mathbf{u}, \boldsymbol{\sigma}) \in X$ we have

$$
\begin{aligned}
\left(P_{k}(\xi), \xi\right)_{X}=2 r(1-r) \int_{\Omega}\|\nabla \mathbf{u}\|^{2} & +\int_{\Omega}\|\boldsymbol{\sigma}\|^{2}+\mathrm{D} \int_{\Omega}\|\nabla \boldsymbol{\sigma}\|^{2} \\
& +\mathrm{We} \int_{\Omega} g_{a}(\nabla \mathbf{u}, \boldsymbol{\sigma}): \boldsymbol{\sigma}-2 r\langle\mathbf{f}, \mathbf{u}\rangle .
\end{aligned}
$$

From the Hölder inequality and the Sobolev injection $W \subset L^{4}(\Omega)^{9}$, there exists a positive constant $C_{\Omega}$ which only depends on the domain, such that $\|\boldsymbol{\sigma}\|_{L^{4}} \leq C_{\Omega}\|\boldsymbol{\sigma}\|_{W}$. Thus, the contribution $g_{a}$ satisfied

$$
\int_{\Omega} g_{a}(\nabla \mathbf{u}, \boldsymbol{\sigma}): \boldsymbol{\sigma} \leq 2|a| \int_{\Omega}\|\nabla \mathbf{u}\|\|\boldsymbol{\sigma}\|^{2} \leq 2|a|\|\nabla \mathbf{u}\|_{L^{2}}\|\boldsymbol{\sigma}\|_{L^{4}}^{2},
$$

which yields

$$
\int_{\Omega} g_{a}(\nabla \mathbf{u}, \boldsymbol{\sigma}): \boldsymbol{\sigma} \leq 2|a| C_{\Omega}^{2}\|\mathbf{u}\|_{V}\|\boldsymbol{\sigma}\|_{W}^{2}
$$

We deduce that

$$
\begin{aligned}
\left(P_{k}(\xi), \xi\right)_{X} \geq & 2 r(1-r)\|\mathbf{u}\|_{V}^{2}+\min (1, \mathrm{D})\|\boldsymbol{\sigma}\|_{W}^{2} \\
& -2|a| C_{\Omega}^{2} \mathrm{We}\|\mathbf{u}\|_{V}\|\boldsymbol{\sigma}\|_{W}^{2}-2 r\|\mathbf{f}\|_{H^{-1}}\|\mathbf{u}\|_{V}
\end{aligned}
$$

Using the definition of the norm $\|\xi\|_{X}^{2}=2 r\|\mathbf{u}\|_{V}^{2}+\|\boldsymbol{\sigma}\|_{W}^{2}$, we obtain

$$
\left(P_{k}(\xi), \xi\right)_{X} \geq \min (1-r, \mathrm{D})\|\xi\|_{X}^{2}-\frac{\sqrt{2}|a| C_{\Omega}^{2} \mathrm{We}}{\sqrt{r}}\|\xi\|_{X}^{3}-\sqrt{2 r}\|\mathbf{f}\|_{H^{-1}}\|\xi\|_{X} .
$$

We write this inequality as $\left(P_{k}(\xi), \xi\right)_{X} \geq\|\xi\|_{X}\left(-\alpha\|\xi\|_{X}^{2}+\beta\|\xi\|_{X}-\gamma\right)$.

- If $a \neq 0$ (i.e. $\alpha>0$ ), we deduce that $\left(P_{k}(\xi), \xi\right)_{X}$ may be positive for some value of $\xi$ if the discriminant $\beta^{2}-4 \alpha \gamma$ is nonnegative; in this case we have

$$
\left(P_{k}(\xi), \xi\right)_{X} \geq 0 \Longleftrightarrow \frac{\beta-\sqrt{\beta^{2}-4 \alpha \gamma}}{2 \alpha} \leq\|\xi\|_{X} \leq \frac{\beta+\sqrt{\beta^{2}-4 \alpha \gamma}}{2 \alpha} .
$$

In particular, by denoting

$$
C_{(\mathrm{II})}=\frac{\beta-\sqrt{\beta^{2}-4 \alpha \gamma}}{2 \alpha}
$$


we obtain

$$
\|\xi\|_{X}=C_{(\mathrm{II})} \quad \Longrightarrow \quad\left(P_{k}(\xi), \xi\right)_{X} \geq 0 .
$$

We also note that $C_{(\mathrm{II})}>0$ (except for the case $\mathbf{f}=\mathbf{0}($ i.e. $\gamma=0$ ) where we can use instead of $C_{(\mathrm{II})}$ any constant $C$ such that $\left.0<C<\frac{\beta}{\alpha}\right)$.

- If $a=0$ (i.e. $\alpha=0$ ), we easily have

$$
\left(P_{k}(\xi), \xi\right)_{X} \geq 0 \quad\|\xi\|_{X} \geq \frac{\gamma}{\beta}
$$

We then use

$$
C_{(\mathrm{II})}=\frac{\gamma}{\beta}
$$

As for the case $a \neq 0$ we have $C_{(\mathrm{II})}>0$ (except for $\mathbf{f}=\mathbf{0}(i . e . \gamma=0$ ) where we can use instead of $C_{(\mathrm{II})}$ any positive constant).

Finally, assumptions given by Lemma 1 are satisfied taking $R=C_{(\mathrm{II})}$ as soon as $\beta^{2}-4 \alpha \gamma \geq 0$, which is equivalent to the condition $C_{(\mathrm{I})} \leq 1$ introduced in Theorem 1. By Lemma 1, we deduce that, for any $k \in \mathbb{N}$, Equations (4) admit a solution $\left(\mathbf{u}_{k}, \boldsymbol{\sigma}_{k}\right)$ which satisfies

$$
2 r\left\|\mathbf{u}_{k}\right\|_{V}^{2}+\left\|\boldsymbol{\sigma}_{k}\right\|_{W}^{2} \leq C_{(\mathrm{II})}^{2} .
$$

This estimate (7) implies that the sequence $\left(\mathbf{u}_{k}, \boldsymbol{\sigma}_{k}\right)$ remains bounded in $V \times W$. Thus there exists some $(\mathbf{u}, \boldsymbol{\sigma}) \in V \times W$ and a subsequence (still denoted by $k)$ such that $\left(\mathbf{u}_{k}, \boldsymbol{\sigma}_{k}\right) \rightarrow(\mathbf{u}, \boldsymbol{\sigma})$ for the weak topology of $V \times W$, as $k \rightarrow+\infty$. As $H^{1} \subset L^{2}$ with compact injection, $\left(\mathbf{u}_{k}, \boldsymbol{\sigma}_{k}\right) \rightarrow(\mathbf{u}, \boldsymbol{\sigma})$ for the strong topology of $L^{2}(\Omega)^{3} \times L^{2}(\Omega)^{9}$. The consequences are twofold:

- We can pass to the limit in all the terms of Equations (4), and deduce that $(\mathbf{u}, \boldsymbol{\sigma})$ is a solution of $(2)$;

- Due to the usual property of the weak limit, we have

$$
2 r\|\mathbf{u}\|_{V}^{2}+\|\boldsymbol{\sigma}\|_{W}^{2} \leq \liminf _{k \rightarrow+\infty} 2 r\left\|\mathbf{u}_{k}\right\|_{V}^{2}+\left\|\boldsymbol{\sigma}_{k}\right\|_{W}^{2} \leq C_{(\mathrm{II})}^{2}
$$

This concludes the proof of existence of a solution $(\mathbf{u}, \boldsymbol{\sigma})$ of problem (2) satisfying Eq. (3). 
Now let us prove the existence of a pressure field associated to the incompressibility condition. For a solution $(\mathbf{u}, \boldsymbol{\sigma})$ of problem $(2)$, we have, for all $\mathbf{v} \in \mathcal{D}(\Omega)$ such that $\operatorname{div} \mathbf{v}=0$,

$$
\langle\operatorname{Re}(\mathbf{u} \cdot \nabla \mathbf{u})-(1-r) \Delta \mathbf{u}-\operatorname{div} \boldsymbol{\sigma}-\mathbf{f}, \mathbf{v}\rangle=0 .
$$

By De Rham theorem, there exists a pressure $p \in \mathcal{D}^{\prime}(\Omega)$ such that Eq. (1) holds in $\mathcal{D}^{\prime}(\Omega)$. The regularity of $\Delta \mathbf{u}, \mathbf{u} \cdot \nabla \mathbf{u}$, div $\boldsymbol{\sigma}$ and $\mathbf{f}$ implies that the pressure $p$ is more regular. For instance, by Sobolev embeddings, we have

$$
\mathbf{u} \in V \subset L^{6}(\Omega)^{3}
$$

and since $\nabla u \in L^{2}(\Omega)^{3 \times 3}$, then the convective term $\mathbf{u} \cdot \nabla \mathbf{u}$ belongs to $L^{3 / 2}(\Omega)$. Thus, we have

$$
\nabla p=\Delta \mathbf{u}+\operatorname{div} \boldsymbol{\sigma}+\mathbf{f}-\operatorname{Re}(\mathbf{u} \cdot \nabla u) \in H^{-1}(\Omega) .
$$

This implies that $p \in L^{2}(\Omega)$, see $[17, \mathrm{p} .14]$.

\subsection{Proof of uniqueness}

Let $\left(\mathbf{u}_{1}, \boldsymbol{\sigma}_{1}\right)$ and $\left(\mathbf{u}_{2}, \boldsymbol{\sigma}_{2}\right)$ be two solutions of (2) and introduce the difference $(\mathbf{u}, \boldsymbol{\sigma})=\left(\mathbf{u}_{2}, \boldsymbol{\sigma}_{2}\right)-\left(\mathbf{u}_{1}, \boldsymbol{\sigma}_{1}\right)$. By subtraction we obtain, for all $(\mathbf{v}, \boldsymbol{\tau}) \in V \times W$,

$$
\begin{array}{r}
\operatorname{Re} \int_{\Omega}\left(\mathbf{u} \cdot \nabla \mathbf{u}_{2}+\mathbf{u}_{1} \cdot \nabla \mathbf{u}\right) \cdot \mathbf{v}+(1-r) \int_{\Omega} \nabla \mathbf{u}: \nabla \mathbf{v}+\int_{\Omega} \boldsymbol{\sigma}: \mathbb{D}(\mathbf{v})=0, \\
\mathrm{We} \int_{\Omega}\left(\mathbf{u} \cdot \nabla \boldsymbol{\sigma}_{2}+\mathbf{u}_{1} \cdot \nabla \boldsymbol{\sigma}+g_{a}\left(\nabla \mathbf{u}, \boldsymbol{\sigma}_{2}\right)+g_{a}\left(\nabla \mathbf{u}_{1}, \boldsymbol{\sigma}\right)\right): \boldsymbol{\tau}+\int_{\Omega} \boldsymbol{\sigma}: \boldsymbol{\tau} \\
+\mathrm{D} \int_{\Omega} \nabla \boldsymbol{\sigma}: \nabla \boldsymbol{\tau}=2 r \int_{\Omega} \boldsymbol{\tau}: \mathbb{D}(\mathbf{u}) .
\end{array}
$$

Taking $(\mathbf{v}, \boldsymbol{\tau})=(2 r \mathbf{u}, \boldsymbol{\sigma})$ and adding the two equations, we obtain

$$
\begin{aligned}
2 r(1-r)\|\mathbf{u}\|_{V}^{2}+\min (1, \mathrm{D})\|\boldsymbol{\sigma}\|_{W}^{2} \leq-2 r \operatorname{Re} \int_{\Omega}\left(\mathbf{u} \cdot \nabla \mathbf{u}_{2}\right) \cdot \mathbf{u} \\
\quad-\mathrm{We} \int_{\Omega}\left(\mathbf{u} \cdot \nabla \boldsymbol{\sigma}_{2}+g_{a}\left(\nabla \mathbf{u}, \boldsymbol{\sigma}_{2}\right)+g_{a}\left(\nabla \mathbf{u}_{1}, \boldsymbol{\sigma}\right)\right): \boldsymbol{\sigma} .
\end{aligned}
$$

By definition of the bilinear function $g_{a}$, we have

$$
\int_{\Omega} g_{a}\left(\nabla \mathbf{u}, \boldsymbol{\sigma}_{2}\right): \boldsymbol{\sigma}=(a+1) \int_{\Omega}\left(\nabla \mathbf{u} \cdot \boldsymbol{\sigma}_{2}\right): \boldsymbol{\sigma}+(a-1) \int_{\Omega}\left(\boldsymbol{\sigma}_{2} \cdot \nabla \mathbf{u}\right): \boldsymbol{\sigma} .
$$


Using the Hölder inequality and the Sobolev injection $W \subset L^{4}(\Omega)^{9}$ we deduce that

$$
\left|\int_{\Omega} g_{a}\left(\nabla \mathbf{u}, \boldsymbol{\sigma}_{2}\right): \boldsymbol{\sigma}\right| \leq 2 C_{\Omega}^{2}\|\mathbf{u}\|_{V}\left\|\boldsymbol{\sigma}_{2}\right\|_{W}\|\boldsymbol{\sigma}\|_{W} .
$$

Estimating the other terms as in Equation (6), we obtain

$$
\begin{aligned}
2 r(1-r)\|\mathbf{u}\|_{V}^{2}+\min (1, \mathrm{D})\|\boldsymbol{\sigma}\|_{W}^{2} \leq & 2 r C_{\Omega}^{2} \operatorname{Re}\left\|\mathbf{u}_{2}\right\|_{V}\|\mathbf{u}\|_{V}^{2} \\
& +3 C_{\Omega}^{2} \mathrm{We}\left\|\boldsymbol{\sigma}_{2}\right\|_{W}\|\mathbf{u}\|_{V}\|\boldsymbol{\sigma}\|_{W} \\
& +2|a| C_{\Omega}^{2} \mathrm{We}\left\|\mathbf{u}_{1}\right\|_{V}\|\boldsymbol{\sigma}\|_{W}^{2} .
\end{aligned}
$$

As the solutions $\left(\mathbf{u}_{1}, \boldsymbol{\sigma}_{1}\right)$ and $\left(\mathbf{u}_{2}, \boldsymbol{\sigma}_{2}\right)$ satisfy Equation (3), we deduce that

$$
\begin{aligned}
2 r(1-r)\|\mathbf{u}\|_{V}^{2}+\min (1, \mathrm{D})\|\boldsymbol{\sigma}\|_{W}^{2} \leq \frac{C_{\Omega}^{2} C_{(\mathrm{II})}}{\sqrt{2 r}} & \left(2 r \operatorname{Re}\|\mathbf{u}\|_{V}^{2}\right. \\
& +3 \mathrm{We} \sqrt{2 r}\|\mathbf{u}\|_{V}\|\boldsymbol{\sigma}\|_{W} \\
& \left.+2|a| \mathrm{We}\|\boldsymbol{\sigma}\|_{W}^{2}\right) .
\end{aligned}
$$

From Young inequality we deduce

$$
3 \mathrm{We} \sqrt{2 r}\|\mathbf{u}\|_{V}\|\boldsymbol{\sigma}\|_{W} \leq 2 r \operatorname{Re}\|\mathbf{u}\|_{V}^{2}+\frac{9 \mathrm{We}^{2}}{4 \operatorname{Re}}\|\boldsymbol{\sigma}\|_{W}^{2}
$$

Consequently, Estimate (8) reads $2 r A\|\mathbf{u}\|_{V}^{2}+B\|\boldsymbol{\sigma}\|_{W}^{2} \leq 0$ where $A$ and $B$ are given by

$$
\begin{aligned}
& A=(1-r)-\frac{4 \operatorname{Re} C_{\Omega}^{2} C_{(\mathrm{II})}}{\sqrt{2 r}} \\
& B=\min (1, \mathrm{D})-\frac{C_{\Omega}^{2} C_{(\mathrm{II})}}{\sqrt{2 r}}\left(\frac{9 \mathrm{We}^{2}}{4 \mathrm{Re}}+2|a| \mathrm{We}\right) .
\end{aligned}
$$

Using the fact that $C_{(\mathrm{II})}$ tends to 0 as $\|\mathbf{f}\|_{H^{-1}}$ tends to 0 , we deduce that for $\|\mathbf{f}\|_{H^{-1}}$ small enough, the coefficients $A$ and $B$ are positive. In the same way, if Re is small enough then $A>0$ and, if We is small, then $B>0$. These inequalities imply $\|\mathbf{u}\|_{V}=\|\boldsymbol{\sigma}\|_{W}=0$, which means $\left(\mathbf{u}_{1}, \boldsymbol{\sigma}_{1}\right)=\left(\mathbf{u}_{2}, \boldsymbol{\sigma}_{2}\right)$.

\subsection{Strong solutions}

As previously, we notice that $\mathbf{u} \in L^{6}(\Omega)^{3}$, so that $\mathbf{u} \cdot \nabla \mathbf{u} \in L^{3 / 2}(\Omega)^{3}$. We also have $\operatorname{div} \boldsymbol{\sigma} \in L^{2}(\Omega)^{3}$ and if $\mathbf{f}$ is regular then the regularity of the Stokes 
problem, see [5], implies that

$$
\mathbf{u} \in W^{2,3 / 2}(\Omega)^{3}, \quad p \in W^{1,3 / 2}(\Omega) .
$$

In the same way, $\mathbf{u} \cdot \nabla \boldsymbol{\sigma}$ and $g_{a}(\nabla \mathbf{u}, \boldsymbol{\sigma})$ belong to $L^{3 / 2}(\Omega)^{3 \times 3}$. The regularity of the Laplace problem implies that

$$
\boldsymbol{\sigma} \in W^{2,3 / 2}(\Omega)^{3 \times 3}
$$

Repeating such a process, we find that $\mathbf{u} \in W^{s, 3 / 2}(\Omega)^{3}, p \in W^{s-1,3 / 2}(\Omega)$ and $\boldsymbol{\sigma} \in W^{s, 3 / 2}(\Omega)^{3 \times 3}$ for any $s \in \mathbb{N}$. The proof is concluded.

\section{Numerical investigation of the vanishing dif- fusion process}

Numerical simulations have been performed in order to illustrate the influence of the diffusive parameter on the stationary solution. After giving some comprehensive details on the numerical method, we will present two simulations:

- The flow past a cylinder in a plane channel is a benchmark case from the literature. The vanishing diffusion process is faced with its formal limit. Let us recall that the non-diffusive model investigated by M. Renardy [16] deals with Dirichlet boundary conditions for the velocity field with an additional assumption: the normal component of the velocity is zero. Thus, no fluid enters or exits the domain, avoiding possible compatibility problems with boundary conditions on the stress tensor, which do not need to be imposed in this particular situation. In the diffusive model, there is no such limitation on the Dirichlet boundary condition. The benchmark case allows us to investigate the convergence of the solution with diffusion to the solution without diffusion, relaxing the assumption on the boundary condition.

- The driven cavity is considered. In this problem, the normal component of the velocity is zero but the discontinuity of the prescribed velocity field at the corners of the domain lead to a loss of regularity of the solution. Therefore, strong solutions do not exist and the framework given by M. Renardy is not valid anymore. Nevertheless, the finite 
element solution is well-defined (for both the diffusive case and the non-diffusive case) and, thus, we investigate the convergence of the solution with diffusion to the solution without diffusion, despite the lack of regularity of the solutions.

The numerical simulations described in Section 3 have been led in the corotationnal case, i.e. $a=0$. The Johnson-Segalman issue is addressed in the concluding remarks in Section 4.

\subsection{Algorithm}

The numerical method is based on the transient version of the Oldroyd model: stationary states are attained as the long-time solution of the evolutive model. If the solution converges in time, then a stationary state is obtained through this process. Note also that for $a=0$ existence of a stationary solution is ensured and, if the data are sufficiently small, the stationary solution is unique. As a consequence, in a non-uniqueness regime, if the algorithm converges, then it is likely to select a stationary solution among all the possible ones.

In order to describe the application of the characteristics method for the particular convective terms in the viscous and elastic equations, we consider the artificial time dependent velocity field $\mathbf{u}(t, \mathbf{x})=\mathbf{u}$ and an artificial time dependence in all involved functions, by introducing the notation $\bar{\phi}(t, \mathbf{x})=$ $\phi(\mathbf{x})$. By convenience, we immediatly drop he overscripts - . Then, we can introduce the following material (or total derivative) operator associated to the velocity field $\mathbf{u}$ :

$$
\frac{\mathrm{D}}{\mathrm{D} t}=\frac{\partial}{\partial t}+\mathbf{u} \cdot \nabla
$$

In this way, the initial equations take the following form.

- the momentum equation can be written as

$$
\operatorname{Re} \frac{\mathrm{D} \mathbf{u}}{\mathrm{D} t}-(1-r) \Delta \mathbf{u}+\nabla p=\operatorname{div} \boldsymbol{\sigma}+\mathbf{f} ;
$$

- the incompressibility equation is unchanged :

$$
\operatorname{div} \mathbf{u}=0
$$

- the constitutive equation can be written as

$$
\mathrm{We}\left(\frac{\mathrm{D} \boldsymbol{\sigma}}{\mathrm{D} t}+g_{a}(\nabla \mathbf{u}, \boldsymbol{\sigma})\right)+\boldsymbol{\sigma}-\mathrm{D} \Delta \boldsymbol{\sigma}=2 r \mathbb{D}(\mathbf{u}) .
$$


Next, by approximating the total derivative by the characteristics method, the previous equations can be approximated by

- momentum equation:

$$
\operatorname{Re} \frac{\mathbf{u}^{n+1}-\mathbf{u}^{n} \circ \kappa^{n}}{\Delta t}-(1-r) \Delta \mathbf{u}^{n+1}+\nabla p^{n+1}=\operatorname{div} \boldsymbol{\sigma}^{n+1}+\mathbf{f}^{n+1} ;
$$

- incompressibility equation:

$$
\operatorname{div} \mathbf{u}^{n+1}=0
$$

- constitutive equation:

$$
\mathrm{We}\left(\frac{\boldsymbol{\sigma}^{n+1}-\boldsymbol{\sigma}^{n} \circ \kappa^{n}}{\Delta t}+g_{a}\left(\nabla \mathbf{u}^{n+1}, \boldsymbol{\sigma}^{n+1}\right)\right)+\boldsymbol{\sigma}^{n+1}-\mathrm{D} \Delta \boldsymbol{\sigma}^{n+1}=2 r \mathbb{D}\left(\mathbf{u}^{n+1}\right) \text {, }
$$

where the index $n+1$ denotes the approximation at the artificial time $t^{n+1}$ of the introduced time dependent functions and $\Delta t$ denotes an artificial time step. Moreover, the function $\kappa^{n}$ is defined at each spatial point of the domain by $\kappa^{n}(\mathbf{x})=\kappa\left(t^{n+1}, \mathbf{x} ; t^{n}\right)$, that denotes the position at time $t^{n}$ of the point placed in $\mathbf{x}$ at time $t^{n+1}$ and moving along the integral path (characteristic curve) defined by the velocity field $\mathbf{u}$, so that $\kappa^{n}(\mathbf{x})$ can be obtained from the solution of the final value ODE problem:

$$
\frac{\mathrm{d} \kappa}{\mathrm{d} \tau}\left(t^{n+1}, \mathbf{x} ; \tau\right)=\mathbf{u}\left(\tau, \kappa\left(t^{n+1}, \mathbf{x} ; \tau\right)\right), \quad \kappa\left(t^{n+1}, \mathbf{x} ; t^{n+1}\right)=\mathbf{x} .
$$

In order to pose an approximated problem (which is actually dependent on the parameter $\Delta t$ ), The semi-discretized problem is completed with the boundary conditions now written in terms of $\mathbf{u}^{n+1}$ and $\boldsymbol{\sigma}^{n+1}$, instead of $\mathbf{u}$ and $\boldsymbol{\sigma}$ and, also, some initial condition for both the velocity field and the elastic constraint. In this way, a stationary solution of the Oldroyd model with diffusive stress is obtained by letting $t$ tend to $\infty$.

Before describing in details the effective problems to solve, let us highlight the fact that the discretized problem is strongly coupled. As we are only interested in the long time asymptotic solution, a very simple trick for easier computations consists in splitting the coupling between the velocity field and the elastic stress. In other words, we consider an explicit term in the 
source term of the viscous equation instead of an explicit one: $\operatorname{div} \boldsymbol{\sigma}^{n+1}$ is replaced by $\operatorname{div} \boldsymbol{\sigma}^{n}$. Besides, in order to use classical approximation spaces, the incompressibility condition is treated by duality. Thus, the effective problems to solve, at each time step $t^{n+1}$, now reads

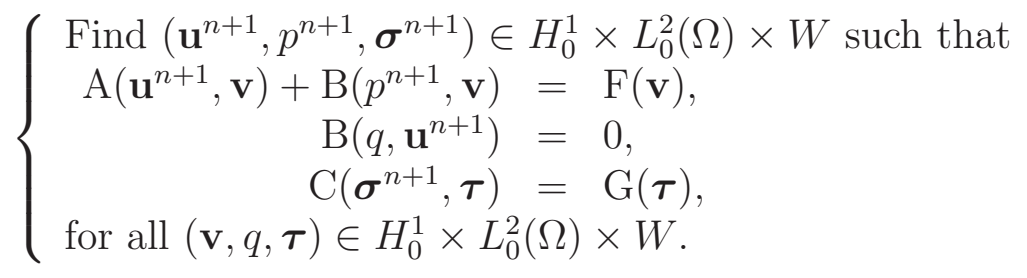

with the following operators

$$
\begin{aligned}
& \mathrm{A}(\mathbf{u}, \mathbf{v}):= \frac{\mathrm{Re}}{\Delta t} \int_{\Omega} \mathbf{u} \cdot \mathbf{v}+2(1-r) \int_{\Omega} \mathbb{D}(\mathbf{u}): \mathbb{D}(\mathbf{v}), \\
& \mathrm{B}(q, \mathbf{u}):=-\int_{\Omega} q \operatorname{div}(\mathbf{u}), \\
& \mathrm{C}(\boldsymbol{\sigma}, \boldsymbol{\tau}):=\frac{\mathrm{We}}{\Delta t} \int_{\Omega} \boldsymbol{\sigma}: \boldsymbol{\tau}+\mathrm{We} \int_{\Omega} g_{a}\left(\nabla \mathbf{u}^{n+1}, \boldsymbol{\sigma}\right): \boldsymbol{\tau} \\
& \quad+\int_{\Omega} \boldsymbol{\sigma}: \boldsymbol{\tau}+\mathrm{D} \int_{\Omega} \nabla \boldsymbol{\sigma}: \nabla \boldsymbol{\tau},
\end{aligned}
$$

and the source terms defined as

$$
\begin{aligned}
\mathrm{F}(\mathbf{v}) & :=-\int_{\Omega} \boldsymbol{\sigma}^{n}: \nabla \mathbf{v}+\frac{\mathrm{Re}}{\Delta t} \int_{\Omega}\left(\mathbf{u}^{n} \circ \kappa^{n}\right) \cdot \mathbf{v}+\left\langle\mathbf{f}^{n+1}, \mathbf{v}\right\rangle, \\
\mathrm{G}(\boldsymbol{\tau}) & :=\frac{\mathrm{We}}{\Delta t} \int_{\Omega}\left(\boldsymbol{\sigma}^{n} \circ \kappa^{n}\right): \boldsymbol{\tau}+2 r \int_{\Omega} \boldsymbol{\tau}: \mathbb{D}\left(\mathbf{u}^{n+1}\right) .
\end{aligned}
$$

Now we have to deal with the spatial discretization of the weak formulation. For this, we consider a triangular mesh of the domain $\Omega$. The first two equations may be analyzed as a Stokes system which is, therefore, treated with $\mathrm{P}_{1}^{+}-\mathrm{P}_{1}$ or $\mathrm{P}_{2}-\mathrm{P}_{1}$ finite elements (for the velocity and pressure fields), in order to ensure the inf-sup condition at the discrete level. When dealing with a diffusive term $\mathrm{D}>0$, the Oldroyd equation is treated with $\mathrm{P}_{1}$ finite elements which naturally approximates $H^{1}(\Omega)$. For comparison needs, when computing the solution without diffusion $\mathrm{D}=0$, the Oldroyd equation is treated with $\mathrm{P}_{0}$ finite elements which naturally approximates $L^{2}(\Omega)$ corresponding to the regularity of the stress tensor. 


\subsection{Flow past a cylinder in a plane channel in 2D}

\subsubsection{Numerical profiles}

As a benchmark case, we consider a viscoelastic flow past a cylinder in a plane channel. The cylinder ensures the fact that classical Poiseuille profiles are not a solution of the system in the viscoelastic case. This case was studied experimentally in [18], and computationally in $[1,8]$ and recently in [9].

The channel-with-cylinder geometry is built upon a channel with length $L=40 R$, height $H=4 R$. Introducing Cartesian ccordinates, the channel is thus described by $x \in(0, L), z \in(-2 R, 2 R)$. The cylinder has a radius $R$ and its center of mass is located at $x=10 R$ and $z=0$, preserving geometric symmetry with respect to the line $\{z=0\}$. No-slip boundary conditions are imposed at $\{z= \pm 2 R\}$ and a parabolic (Poiseuille) velocity profile is imposed at the inlet $\{x=0\}$ and at the outlet $\{x=L\}$ : it is characterized either by the maximal velocity value $U_{\max }$ or the input/output flux $Q$. In all the computations, $R$ has been set to 0.25 and the flux has been set to $Q=1.0$ which corresponds to a maximal velocity $U_{\max }=2 Q /(3 R)=8 / 3$ at the inlet/outlet.

Considering the benchmark case studied in [9], numerical simulations have been performed with

$$
\operatorname{Re}=0.67, \quad \text { We }=1.0, \quad r=0.6, \quad a=0
$$

and different values of D. Note that the focus on the corotational model (i.e. $a=0$ ) guarantees the existence of a stationary solution for both the diffusive and standard models. The study of the Johnson-Segalman (i.e. $a \neq 0$ ) model will be discussed in concluding remarks.

Figure 1 represents the solution of the stationary Oldroyd model without diffusion, near the cylinder, computed with an unstructured mesh made of 136879 triangles and 69342 vertices. This solution is the reference solution when investigating the behaviour of the model with respect to the diffusive parameter.

\subsubsection{Error analysis}

Figure 2 illustrates the influence of the diffusion parameter $\mathrm{D}$ on the velocity field and the elastic stress tensor, as it tends to 0 . The benchmark case 


\begin{tabular}{c|cc|cc}
$\mathrm{D}$ & $\left\|\mathbf{u}^{(\mathrm{D})}-\mathbf{u}^{(0)}\right\|_{L^{2}}$ & $\left\|\mathbf{u}^{(\mathrm{D})}-\mathbf{u}^{(0)}\right\|_{H^{1}}$ & $\left\|\boldsymbol{\sigma}^{(\mathrm{D})}-\boldsymbol{\sigma}^{(0)}\right\|_{L^{2}}$ & $\left\|\boldsymbol{\sigma}^{(\mathrm{D})}-\boldsymbol{\sigma}^{(0)}\right\|_{H^{1}}$ \\
\hline $1 \mathrm{e}-04$ & $3.2 \mathrm{e}-3$ & $1.6 \mathrm{e}-1$ & $2.2 \mathrm{e}-1$ & $6.6 \mathrm{e}+1$ \\
$1 \mathrm{e}-05$ & $8.1 \mathrm{e}-4$ & $5.2 \mathrm{e}-2$ & $1.8 \mathrm{e}-1$ & $63 \mathrm{e}+1$ \\
$1 \mathrm{e}-06$ & $3.4 \mathrm{e}-4$ & $2.3 \mathrm{e}-2$ & $9.9 \mathrm{e}-2$ & $3.5 \mathrm{e}+1$ \\
$1 \mathrm{e}-07$ & $4.9 \mathrm{e}-5$ & $3.3 \mathrm{e}-3$ & $1.4 \mathrm{e}-2$ & $5.1 \mathrm{e}+0$ \\
$1 \mathrm{e}-08$ & $5.1 \mathrm{e}-6$ & $3.4 \mathrm{e}-4$ & $1.5 \mathrm{e}-3$ & $5.3 \mathrm{e}-1$ \\
$1 \mathrm{e}-09$ & $5.4 \mathrm{e}-7$ & $3.4 \mathrm{e}-5$ & $1.5 \mathrm{e}-4$ & $5.3 \mathrm{e}-2$ \\
$1 \mathrm{e}-10$ & $1.0 \mathrm{e}-7$ & $4.5 \mathrm{e}-6$ & $1.5 \mathrm{e}-5$ & $5.3 \mathrm{e}-3$
\end{tabular}

Table 1: Flow past a cylinder in a plane channel in 2D: computation of the error with respect to the diffusive parameter for the velocity and the elastic constraint, in the $L^{2}$-norm and $H^{1}$-norm.

evidences the convergence of the solution corresponding to the diffusive model to the solution of the non-diffusive model, in classical $L^{2}-$ and $H^{1}$-norms (see Table 1). Rates of convergence are numerically derived (see Figure 2).

Linear regressions have been addressed for small values of D (see Figure 2). It shows that the error in the $L^{2}$-norm and the $H^{1}$-norm behaves at order 1 for both the velocity and the elastic constraint.

We point out the fact that the previous observations related to the order of approximation are still valid in the non-inertial regime (e.g. $\operatorname{Re}=0.0$, $r=0.5$, We $=1.0$ ) or inertial regime (e.g. $\mathrm{Re}=10.0, r=0.5$, We $=1.0$ ).

Let us highlight the fact that one could expect that difficulties arise in the vinicity of boundaries identified as inlets, i.e. where the fluid enters the domain. Indeed Dirichlet boundary conditions on the velocity field, at the inlet, enforce the information on the stress tensor to propagate inside the fluid domain because of the transport process. As the stress tensor is transported from the inlet into the fluid domain, Neumann boundary conditions on the elastic stress tensor do not seem to be the most relevant choice in terms of numerical stability. However, numerical simulations do not reveal any instability or boundary layer phenomenon. Let us notice that other boundaries (outlets corresponding to velocity fields expressing the fact that the fluid goes outside the domain, and lateral boundaries corresponding to zero velocity field) are not identified as a source of problems, which is also confirmed by numerical simulations. 


\subsection{Driven cavity in $2 \mathrm{D}$}

\subsubsection{Numerical profiles}

The driven cavity problem is considered. The fluid domain is a unit square. Boundary conditions on the velocity field are prescribed: $\mathbf{u}=\mathbf{0}$ is imposed on lateral and bottom boundaries whereas $\mathbf{u}=(-1,0)$ is imposed on the top boundary. The interest of this problem relies on the loss of regularity of the velocity field, even in the case of the linear Stokes problem: the solution does not belong to $H^{1}(\Omega)$ anymore, because of the discontinuity of the Dirichlet boundary conditions located at the corners of the geometry [4]. Nevertheless, the finite element solution is well defined. Therefore, we observe the behaviour of the elastic tensor with respect to the loss of regularity.

Numerical simulations have been performed with

$$
\operatorname{Re}=10.0, \quad \text { We }=1.0, \quad r=0.5, \quad a=0
$$

and different values of D. Simulations evidence several facts: Figure 3 reveals a large vortex whose typical size corresponds to the size of the domain: this is due to the inertial effects. The loss of regularity is observed on the pressure profiles: singularities are revealed at the top corners of the domain, at which extremal values are reached. Besides, the influence of the diffusion on the tensor profiles is important, playing the role of a regularizing effect, as it is expected, see Figures 4 and 5. Furthermore, velocity profiles are little affected by the diffusion parameter $\mathrm{D}$, as long as it is small enough (less than $10^{-3}$ ). In the end, the driven cavity test suggests that the loss of regularity does not affect the convergence of the solution towards the solution of the Oldroyd problem without diffusion.

\subsubsection{Error analysis}

The numerical tests have been led for various values of the Reynolds number Re, Weissenberg number We and retardation parameter $r$, see Figures $6-13$. In all cases, the following estimates hold:

$$
\begin{aligned}
\left\|\boldsymbol{\sigma}^{(\mathrm{D})}-\boldsymbol{\sigma}^{(0)}\right\|_{L^{2}} & =\mathcal{O}(\mathrm{D}), \quad\left\|\boldsymbol{\sigma}^{(\mathrm{D})}-\boldsymbol{\sigma}^{(0)}\right\|_{H^{1}}=\mathcal{O}(\mathrm{D}), \\
\left\|\mathbf{u}^{(\mathrm{D})}-\mathbf{u}^{(0)}\right\|_{L^{2}} & =\mathcal{O}(\mathrm{D}), \quad\left\|\mathbf{u}^{(\mathrm{D})}-\mathbf{u}^{(0)}\right\|_{H^{1}}=\mathcal{O}(\mathrm{D}) .
\end{aligned}
$$

Note that even if the velocity fields do not belong to $H^{1}$, the lack of regularity is localized at the corner of the domain. In particular, the loss of regularity 
is the same for both $\mathbf{u}^{(\mathrm{D})}$ and $\mathbf{u}^{(0)}$ so that the difference between the two velocity fields is likely to belong to $H^{1}$. This suggests that, despite the lack of regularity of the solution that prevents us from falling into the scope of the work of Renardy [16], the Oldroyd model is a rigorous limit of the diffusive Oldroyd model as the vanishing diffusive solution converges to the non-diffusive solution in $L^{2}$ and even in $H^{1}$.

\subsection{Driven cavity in 3D}

\subsubsection{Numerical profiles}

The driven cavity problem is considered. The fluid domain is a unit cube. Boundary conditions on the velocity field are prescribed: $\mathbf{u}=\mathbf{0}$ is imposed on lateral and bottom boundaries whereas

$$
\mathbf{u}(x, y, 1)=4 x(1-x) y(1-y) \mathbf{e}_{x}
$$

is imposed on the top boundary. Numerical simulations have been performed with a structured mesh, size $30 \times 30 \times 30$, in the inertial regime $\mathrm{Re}=4.0$, elastic regime $\mathrm{We}=1.0$ and $r=0.5$ and different values of $\mathrm{D}$.

Streamlines associated to the velocity field are shown on Figures 14-16.

\subsubsection{Error analysis}

As in the $2 \mathrm{D}$ case, error analysis in $3 \mathrm{D}$ by means of numerical simulations shows that the solution of the diffusive problem is close to the solution of the non-diffusive problem at order 1 in the $L^{2}$-norm or in the $H^{1}$-norm. More precisely, the behaviour of the error with respect to $\mathrm{D}$ can be described as follows, see Figures 17 and 18:

- Figure 17 or 18 , zone I. For values of D less than $10^{-9}$, the computed error is persistant as the machine precision, when computing the error, has been attained.

- Figure 17 or 18, zone II. The convergence result associated to the asymptotic regime $\mathrm{D} \rightarrow 0$ is ensured by the behaviour of the error corresponding to small values of $\mathrm{D}$, namely $\mathrm{D} \sim 10^{-9}-10^{-3}$. In this range, the magnitude of the order of convergence for both the velocity field and the elastic tensor is identified as being equal to 1 . 
- Figure 17 or 18 , zone III. For values of D greater that $10^{-3}$, which lack physical relevance anyway, the corresponding solution cannot be assumed to be close to the solution of the non-diffusive problem as the diffusive effect on the elastic tensor is predominant over the memory effect.

Hence, the computations suggest that the convergence order with respect to $\mathrm{D}$ does not depend on the dimension of the problem.

\section{Concluding remarks}

As stated in Theorem 1, existence of a stationary solution of the diffusive model is guaranteed if

$$
C_{(\mathrm{I})}:=\frac{8|a| C_{\Omega}^{2} \mathrm{We}\|\mathbf{f}\|_{H^{-1}}}{\min (1-r, \mathrm{D})^{2}} \leq 1 .
$$

Uniqueness of the solution is guaranteed if the source term is small enough or if the Weissenberg and Reynolds numbers are small enough. It is also noted that examples of nonuniqueness exist. They are even the source of wellknown phenomenon like shear banding, see [15]. For example in [11] or in [6] it is shown that for $r>8 / 9$ (and $\mathrm{D}=0$ ), there may be three solutions for the stationary Oldroyd problem, forming in the case of a Couette flow a number of shear bands. Besides, as presented in Section 3, convergence in strong topologies in 2D and 3D has been illustrated on benchmark tests, suggesting that the formal asymptotics related to the vanishing diffusion process may be valid at least in some realistic physical situations.

However, the numerical experiments presented in Section 3 have been led with the corotational case only, i. e. $a=0$, and for moderate Weissenberg numbers. Therefore, it is natural to question the validity of this conjecture for different values of $a$ and $W e$, which is discussed in the two next subsections.

\subsection{On the Johnson-Segalman model}

Before addressing the vanishing viscosity process for the Johnson-Segalman model, we can question whether condition expressed by Eq. (10) is technical or necessary when $a \neq 0$, since the asymptotics only makes sense if the stationary solution is defined for any value of D. The Johnson-Segalman 
model with diffusive constraint was particularly studied in [15] in a cylindrical Couette flow. But in the most general case, i.e. without assumption on the geometry and boundary condition, does existence of a solution depend on a choice of parameters that should belong to a limited range of admissible values in order to satisfy Eq. (10) (or a sharper condition)? Or can the condition expressed in Eq. (10) be weakened or even purely forgotten in order to ensure existence of a sationary solution? Supplementary computations have been led and proven to be helpful in the understanding of the diffusive Johnson-Segalman model:

- Table 2 tends to show that Eq. (10) is certainly necessary: too large values of $|a|$ need to be counterbalanced by large values of $\mathrm{D}$ for the algorithm to succeed in defining a stationary state.

- In both benchmark problems, computations with $a= \pm 0.5$ and $a=$ \pm 1.0 (with large values of $\mathrm{D}$ ) reveal velocity profiles which are quite different from those observed with smaller values of $a$.

- The case $a= \pm 0.1$ is somehow intriguing as the solution with diffusive constraint is defined for every value of D. It suggests that, for small values of $|a|$ Eq. (10) may not be necessary. Alternatively the discretization process (in time and space) of the Oldroyd system may induce some relaxation of the condition on the resulting finite-dimensional Oldroyd system.

We would like to underline that these numerical observations are mostly compatible with theoretical results related to the standard Oldroyd models $[10,12,13]$ : global-in-time solutions exist in the case $a=0$ without any assumption and in the case $a \neq 0$ under smallness assumption on the data ; relaxing this smallness assumption in the Johnson-Segalman model drastically weakens the existence result, as the existence of local-in-time solutions only can be guaranteed. These results, related to the transient version of the standard model, shed a light on both theoretical and numerical results: in our algorithm, stationary states are obtained by letting time tend to $+\infty$, which is possible only if a global-in-time solution of the corresponding transient problem exists. In the vanishing diffusion limit, condition expressed by Eq. (10) may express the degeneracy of the behaviour of solutions with respect to time in the Johnson-Segalman models. 


\subsection{On the high Weissenberg regime}

The high Weissenberg regime does not fall into the range of validity of the study, in particular when considering condition expressed by Eq. (10) that ensures existence of a solution. Therefore, at this stage, well-posedness of the problem with large values of the Weissenberg number is an open problem. However, from the numerical point of view, simulations with the algorithm proposed in this paper failed. We argue two possible directions that may justify this observation: first, ill-posedness of the problem could explain the numerical instabilities that are observed in the simulations ; second, the splitting procedure of the algorithm may be the cause of the instabilities as it tends to segregate the two steps of the computations when the Weissenberg number is large, leading to unphysical oscillations of the solution of the transient model and, in the end, numerical instabilities which prevent us from defining a stationary state.

\section{References}

[1] M.A. Alves, F.T. Pinho, P.J. Oliveira. The flow of viscoelastic fluids past a cylinder: finite-volume high-resolution methods, J. NonNewton. Fluid Mech., 97, 207, 2001.

[2] C. Bardos, A.-Y. LE Roux, J.-C. NÉdÉlec. First order quasilinear equations with boundary conditions, Comm. Partial Differential Equations, 4(9), 1017-1034, 1979.

[3] J. BARRETt, E. SüLI. Existence of global weak solutions to some regularized kinetic models for dilute polymers, Multiscale Model. Simul., 6(2), 506-546, 2007.

[4] Z. CAI, Y. WAng. An error estimate for two-dimensional Stokes driven cavity flow, Math. Comp., 78(266), 771-787, 2009.

[5] L. Cattabriga. Su un problema al contorno relativo al sistema di equazioni di Stokes, Rend. Sem. Mat. Univ. Padova, 31, 308-340, 1961.

[6] F. Boyer, L. Chupin, P. FABrie. Numerical study of viscoelastic mixtures through a Cahn-Hilliard flow model, Eur. J. Mech. B Fluids, 23(5), 759-780, 2004. 
[7] P. Constantin, M. Kliegl. Note on global regularity for twodimensional Oldroyd-B fluids with diffusive stress, Arch. Ration. Mech. Anal. 206(3), 725-740, 2012.

[8] Y. Fan, R.I. TAnner, N. Phan-Thien. Galerkin/least-square finiteelement methods for steady viscoelastic flows, J. Non-Newton. Fluid Mech. 84, 1999.

[9] N. Goyal, J.J. DeRKsen. Direct simulations of spherical particles sedimenting in viscoelastic fluids, J. Non-Newton. Fluid Mech. 183-184, $1-13,2012$.

[10] C. Guillopé, J.-C. Saut. Existence results for the flow of viscoelastic fluids with a differential constitutive law, Nonlinear Anal., 15(9), 849869, 1990.

[11] C. Guillopé, J.-C. Saut. Global existence and one-dimensional nonlinear stability of shearing motions of viscoelastic fluids of Oldroyd type, RAIRO Modél. Math. Anal. Numér., 24(3), 369-401, 1990.

[12] P.-L. Lions, N. Masmoudi. Global solutions for some Oldroyd models of non-Newtonian flows, Chinese Ann. Math. Ser. B, 21(2), 131-146, 2000 .

[13] L. Molinet, R. TAlhouk. On the global and periodic regular flows of viscoelastic fluids with a differential constitutive law, NoDEA Nonlinear Differential Equations Appl., 11(3), 349-359, 2004.

[14] L. Molinet, R. Talhouk. Newtonian limit for weakly viscoelastic fluid flows of Oldroyd type, SIAM J. Math. Anal. 39(5), 1577-1594, 2008.

[15] P. D. Olmsted, O. Radulescu, C.-Y. D. Lu. Johnson-Segalman model with a diffusion term in cylindrical Couette flow, J. Rheol., 44(2), 257-275, 2000.

[16] M. RENARDy. Existence of slow steady flows of viscoelastic fluids with differential constitutive equations, Z. Angew. Math. Mech., 65(9), 449451, 1985.

[17] R. Temam. Navier-Stokes equations. Theory and numerical analysis. Studies in Mathematics and its Applications, Vol. 2, North-Holland Publishing Co., Amsterdam, x+500, 1977. 
[18] J.M. Verhelst, F.T.M. Nieuwstadt. Visco-elastic flow past circular cylinders mounted in a channel: experimental measurements of velocity and drag, J. Non-Newton. Fluid Mech. 116, 301, 2004. 


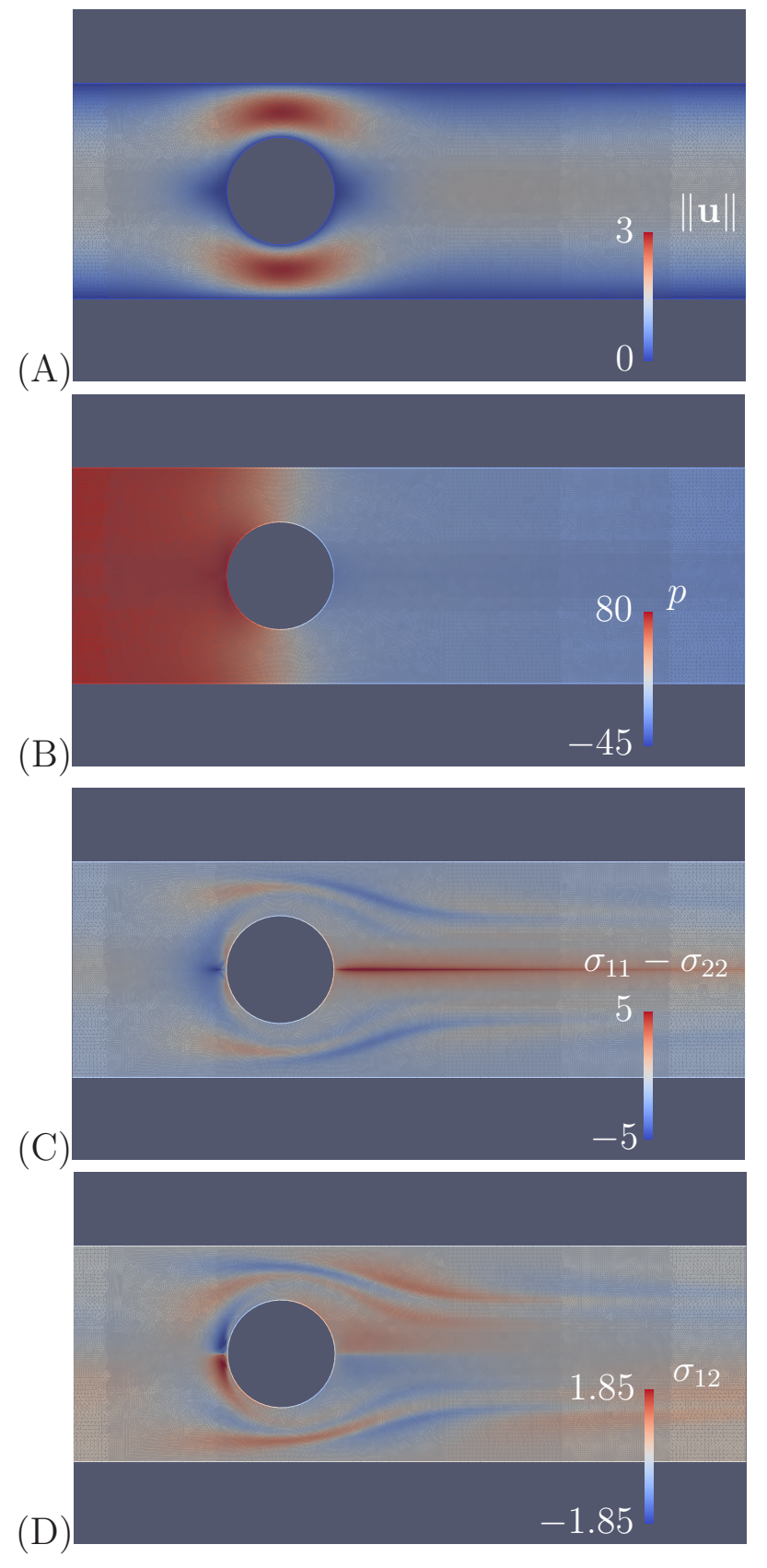

Figure 1: Solution of the stationary Oldroyd model near the cylinder, with $\mathrm{Re}=0.67, r=0.6, a=0, \mathrm{We}=1.0, \mathrm{D}=0.0$. (A): norm of the velocity field, (B): pressure $p,(\mathrm{C})$ : elongational component of the elastic tensor $\sigma_{11}-\sigma_{22}$, (D): shear component of the elastic tensor $\sigma_{12}$. 

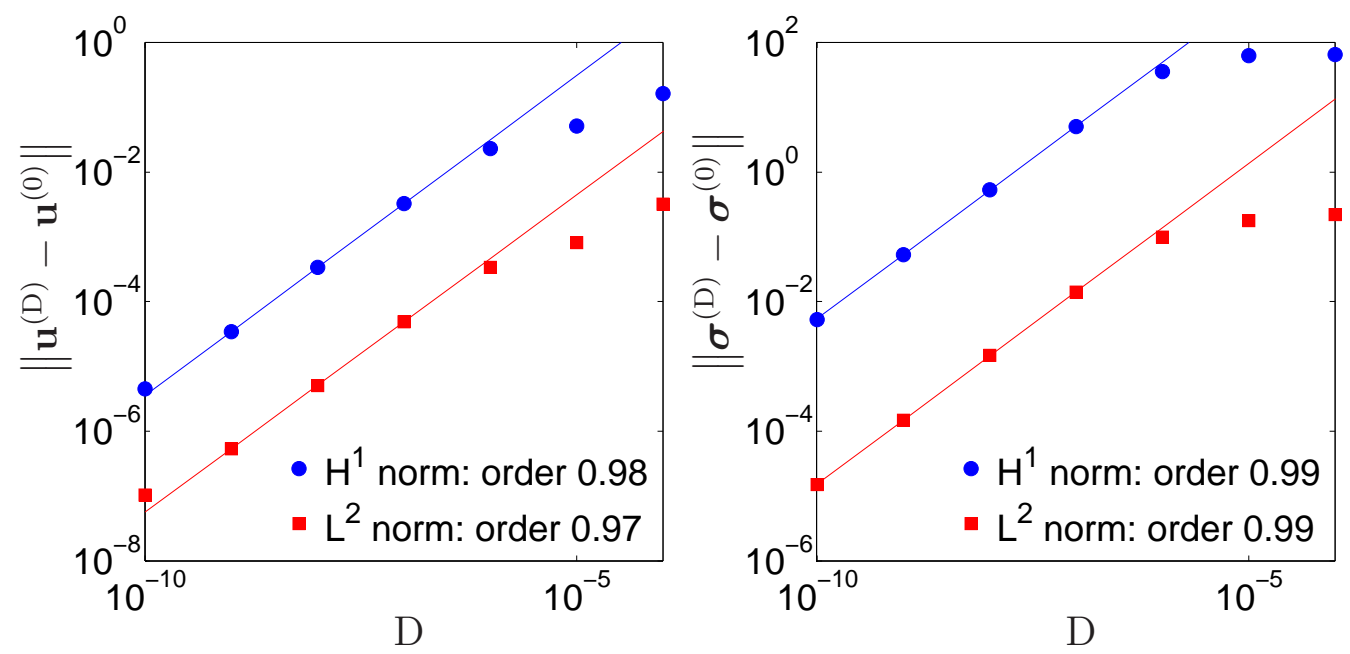

Figure 2: Flow past a cylinder in a plane channel in 2D: influence of the diffusive term on the error on the velocity (1.) and the elastic constraint (r.).

\begin{tabular}{|c|c|c|c|c|c|c|c|c|}
\hline & $\mathrm{D}=10^{+0}$ & $\mathrm{D}=10^{-1}$ & $\mathrm{D}=$ & $0^{-2}$ & $\mathrm{D}=$ & $0^{-3}$ & \multicolumn{2}{|c|}{$\mathrm{D}=0$} \\
\hline & $\begin{array}{lll}(\mathrm{P}) & (\mathrm{C})\end{array}$ & $\overline{(\mathrm{P})}(\mathrm{C})$ & $\overline{(\mathrm{P})}$ & $\overline{(\mathrm{C})}$ & $\overline{(\mathrm{P})}$ & $\overline{(\mathrm{C})}$ & $\overline{(\mathrm{P})}$ & $\overline{(\mathrm{C})}$ \\
\hline$a=+1.0$ & & $\otimes$ & $\nabla$ & $\otimes$ & $\nabla$ & 区 & $\otimes$ & $\nabla$ \\
\hline+0.8 & & & 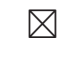 & 凶 & $\bigotimes$ & 凶 & $凶$ & $凶$ \\
\hline+0.5 & & & C & $\mathrm{O}$ & 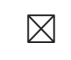 & $凶$ & $凶$ & $凶$ \\
\hline+0.1 & & & & & C & 0 & C & 0 \\
\hline-0.1 & & & & & 0 & 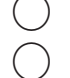 & ( & ? \\
\hline-0.5 & & & 0 & $\mathrm{C}$ & $\bigotimes$ & $\nabla$ & $凶$ & $凶$ \\
\hline-0.8 & & 0 & $\bigotimes$ & 凶 & $凶$ & $凶$ & $\bigotimes$ & $凶$ \\
\hline-1.0 & $\bigcirc$ & $凶$ & $\bigotimes$ & $\bigotimes$ & $凶$ & $凶$ & $凶$ & $凶$ \\
\hline
\end{tabular}

Table 2: Simulations of the Johnson-Segalman model with diffusive constraint for the 2D benchmarks: $(\mathrm{P})$ stands for the benchmark related to the flow past a cylinder in a plane channel and (C) stands for the driven cavity problem. Symbol $\bigcirc$ means that a stationary state has been captured whereas symbol $\otimes$ means that the algorithm has failed in capturing a stationary state. 

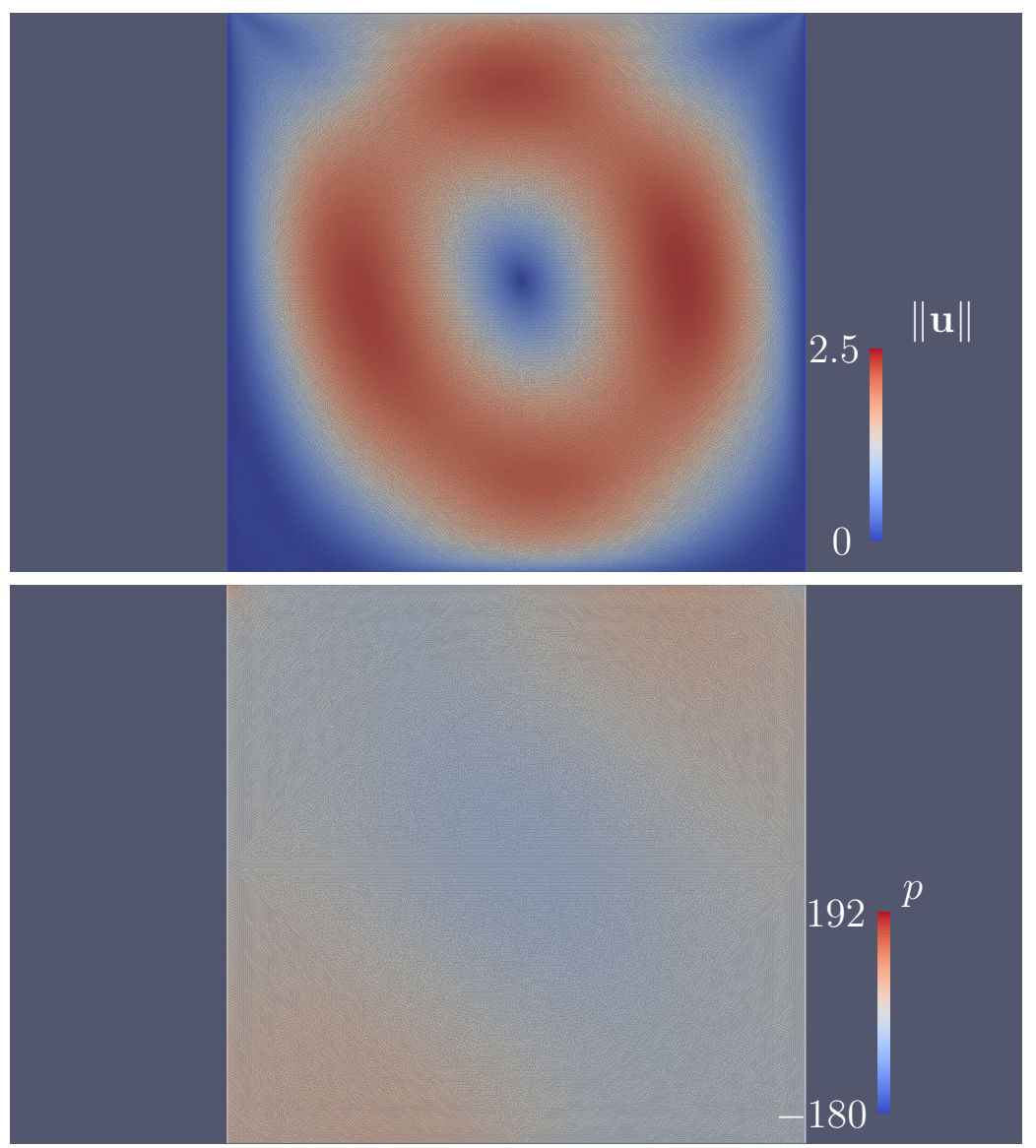

Figure 3: Solution of the stationary Oldroyd model for the driven cavity problem with $\mathrm{Re}=10.0, r=0.5, a=0, \mathrm{We}=1.0, \mathrm{D}=0.0$ : (t.) Norm of the velocity field, (b.) pressure field. 

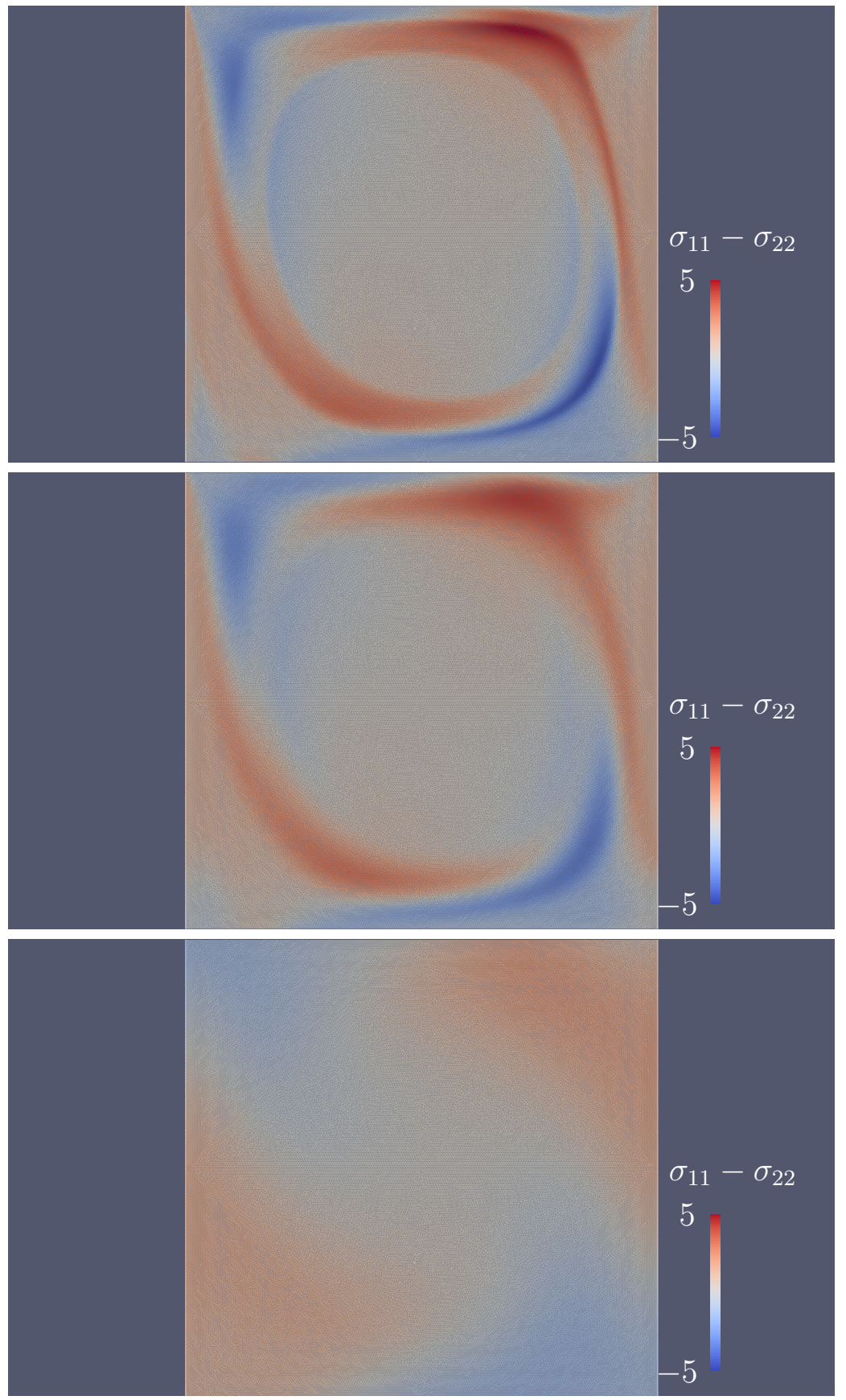

Figure 4: Solution of the stationary Oldroyd model for the driven cavity problem with $\mathrm{Re}=10.0$, We $=1.0, r=0.5, a=0$. Elongational component of the elastic tensor $\sigma_{11}-\sigma_{22}$. (t.): $\mathrm{D}=0.000$, (m.): $\mathrm{D}=0.001$, (b.): $\mathrm{D}=0.100$. 

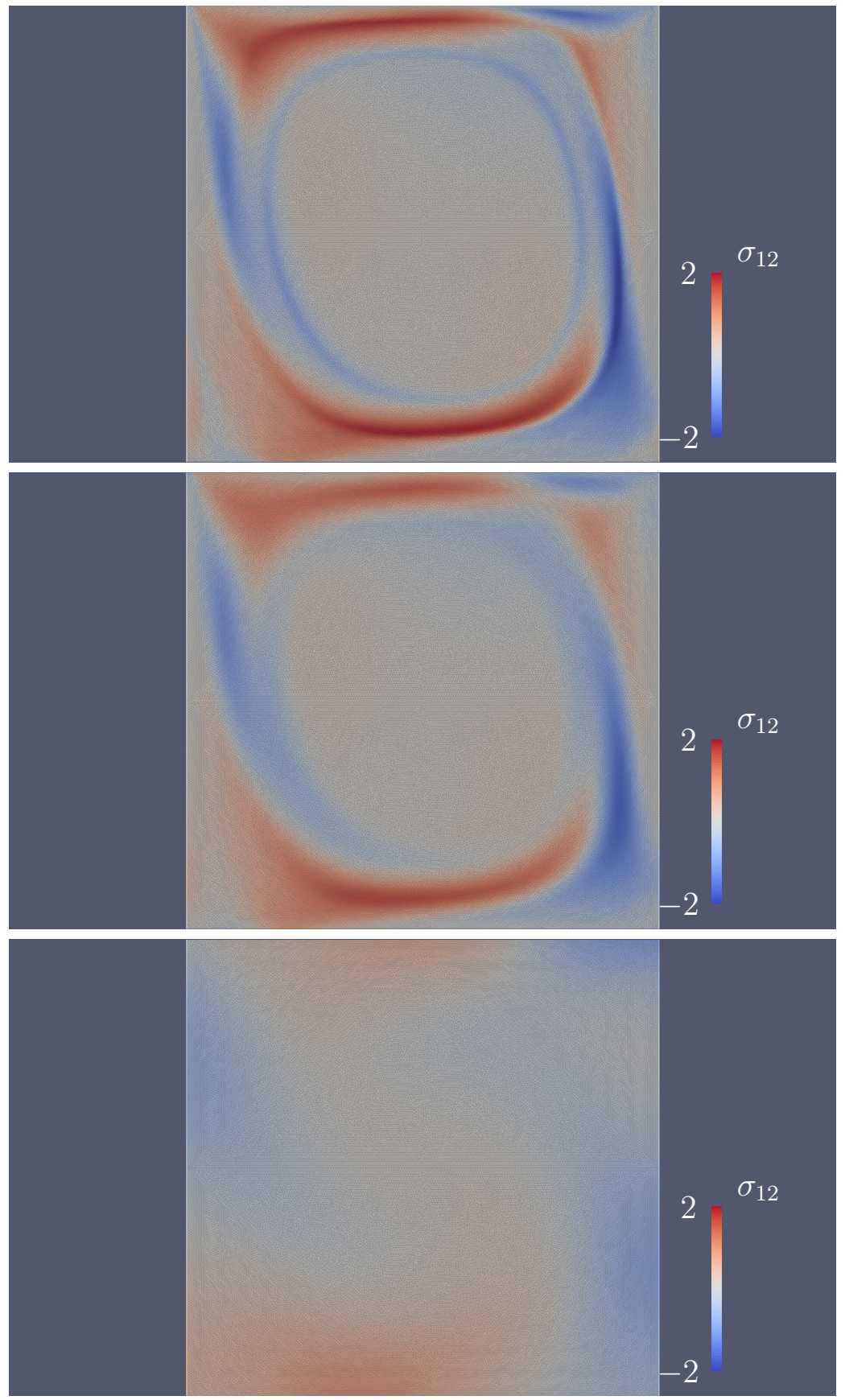

Figure 5: Solution of the stationary Oldroyd model for the driven cavity problem with $\mathrm{Re}=10.0$, We $=1.0, r=0.5, a=0$. Shear component of the elastic tensor $\sigma_{12}$. (t.): $\mathrm{D}=0.000$, (m.): $\mathrm{D}=0.001$, (b.): $\mathrm{D}=0.100$. 

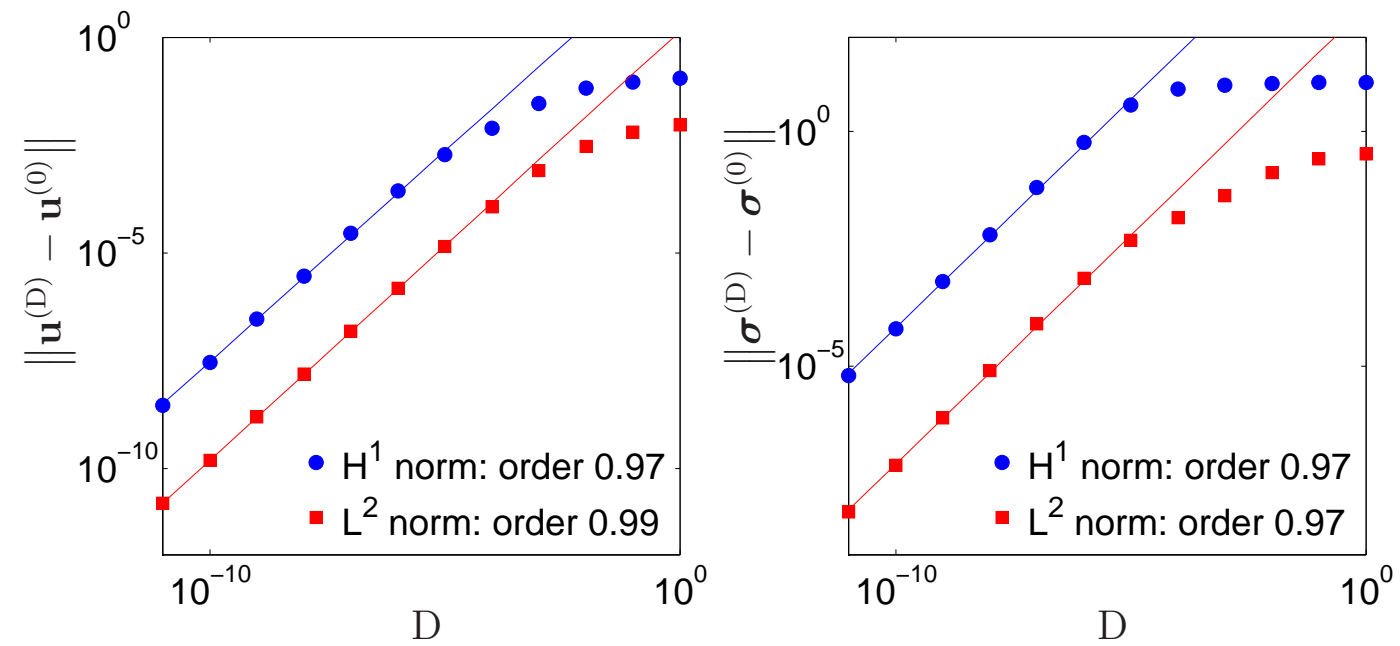

Figure 6: Lid-driven cavity in $2 \mathrm{D}$ with $\mathrm{Re}=0.0, \mathrm{We}=0.5, r=0.2, a=0$ : influence of the diffusive term on the error on the velocity (1.) and the elastic constraint (r.).
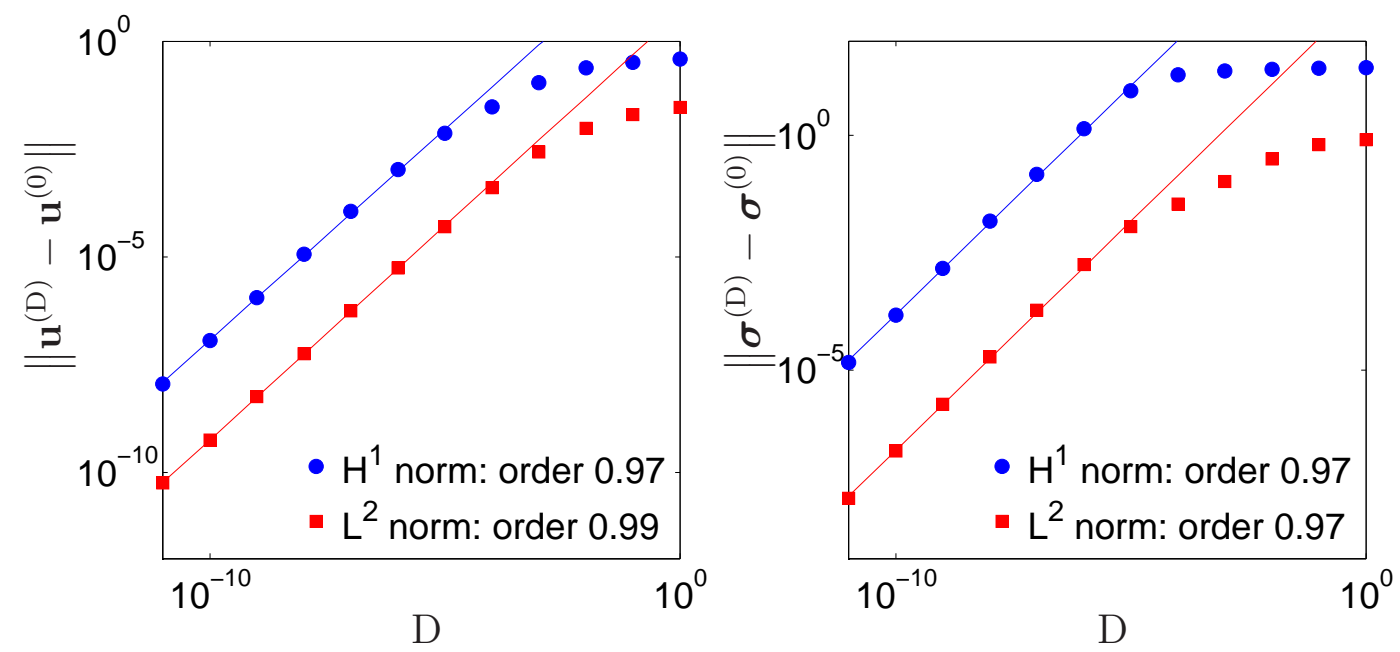

Figure 7: Lid-driven cavity in $2 \mathrm{D}$ with $\mathrm{Re}=0.0, \mathrm{We}=0.5, r=0.5, a=0$ : influence of the diffusive term on the error on the velocity (l.) and the elastic constraint (r.). 

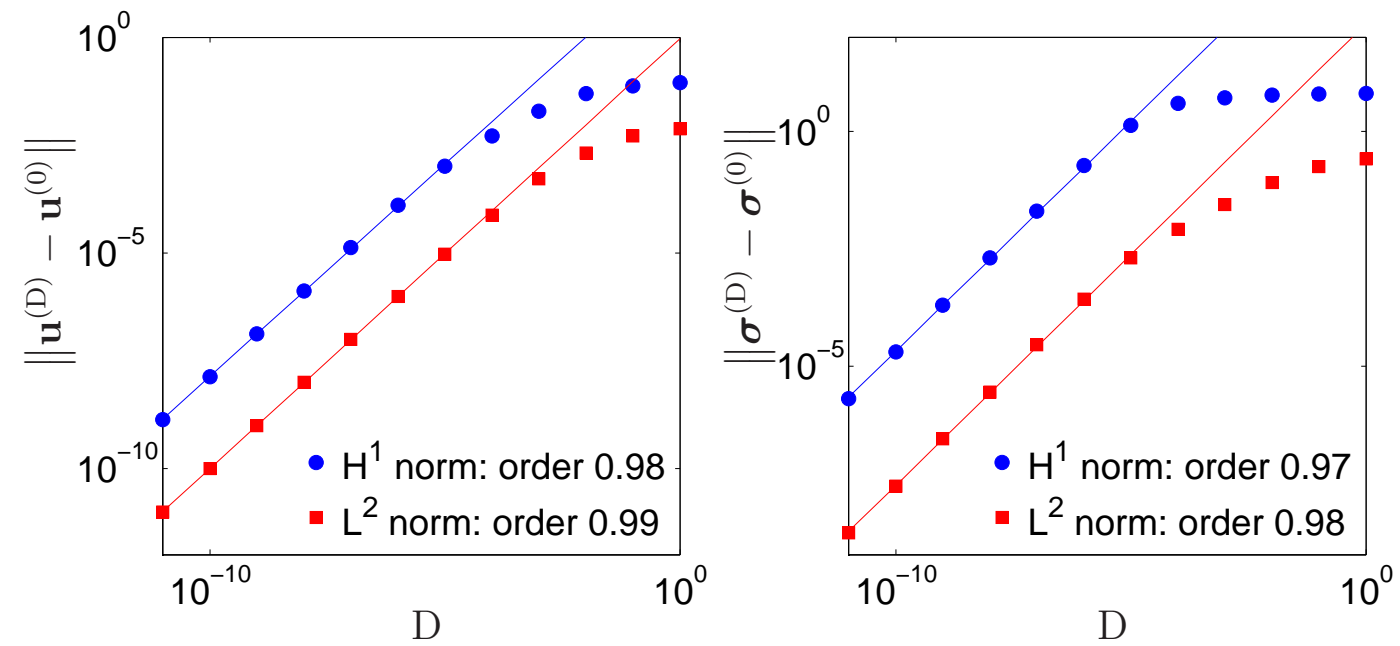

Figure 8: Lid-driven cavity in $2 \mathrm{D}$ with $\mathrm{Re}=0.0, \mathrm{We}=1.0, r=0.2, a=0$ : influence of the diffusive term on the error on the velocity (l.) and the elastic constraint (r.).
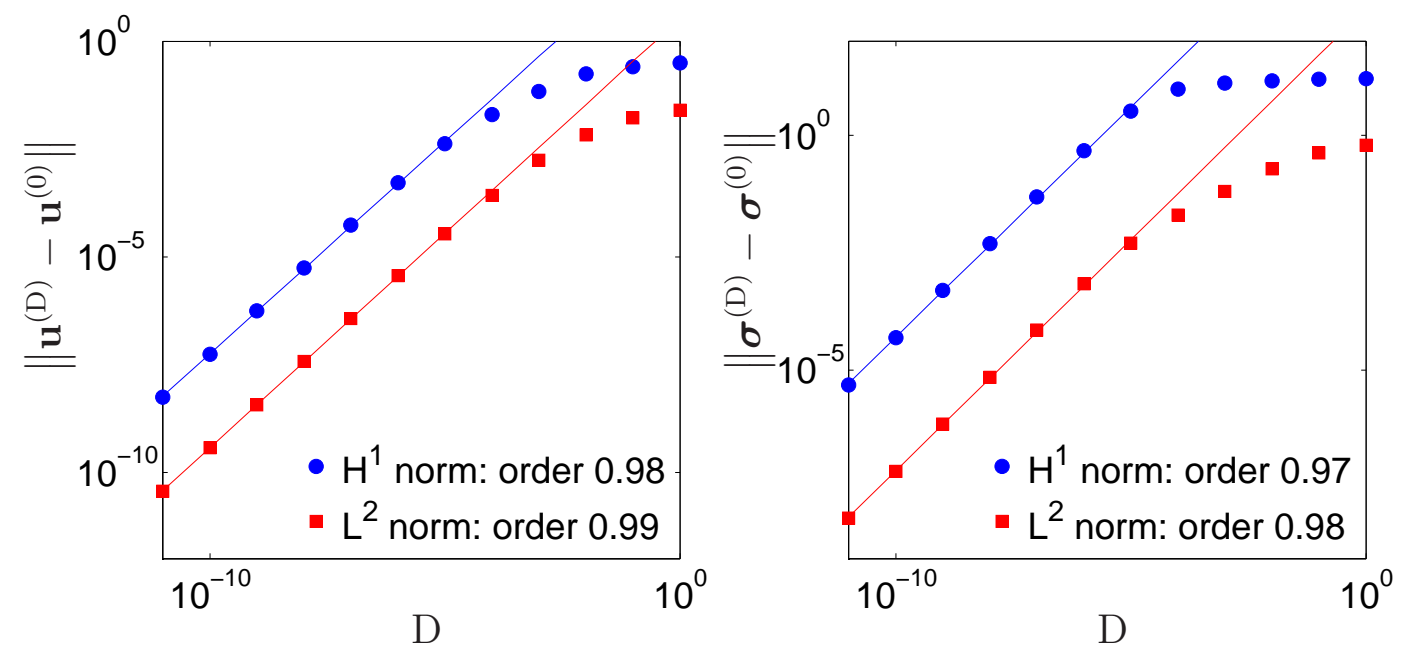

Figure 9: Lid-driven cavity in $2 \mathrm{D}$ with $\mathrm{Re}=0.0, \mathrm{We}=1.0, r=0.5, a=0$ : influence of the diffusive term on the error on the velocity (l.) and the elastic constraint (r.). 

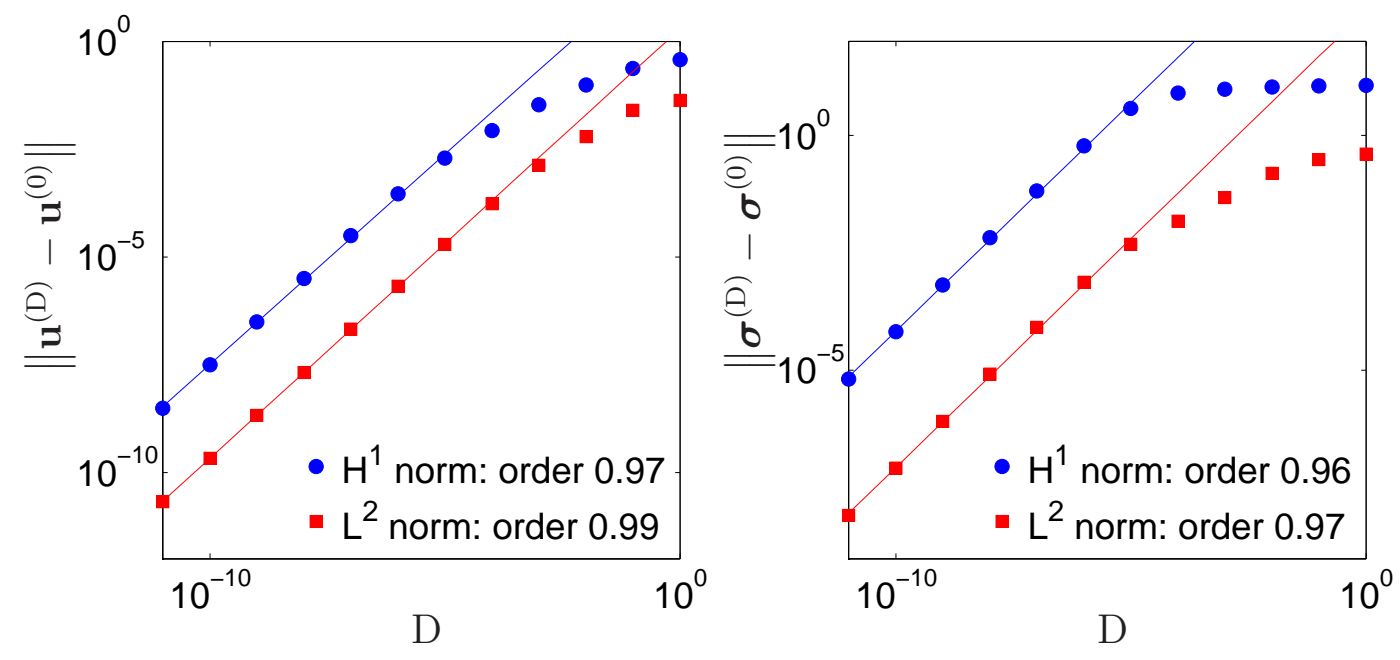

Figure 10: Lid-driven cavity in $2 \mathrm{D}$ with $\mathrm{Re}=5.0, \mathrm{We}=0.5, r=0.2, a=0$ : influence of the diffusive term on the error on the velocity (l.) and the elastic constraint (r.).
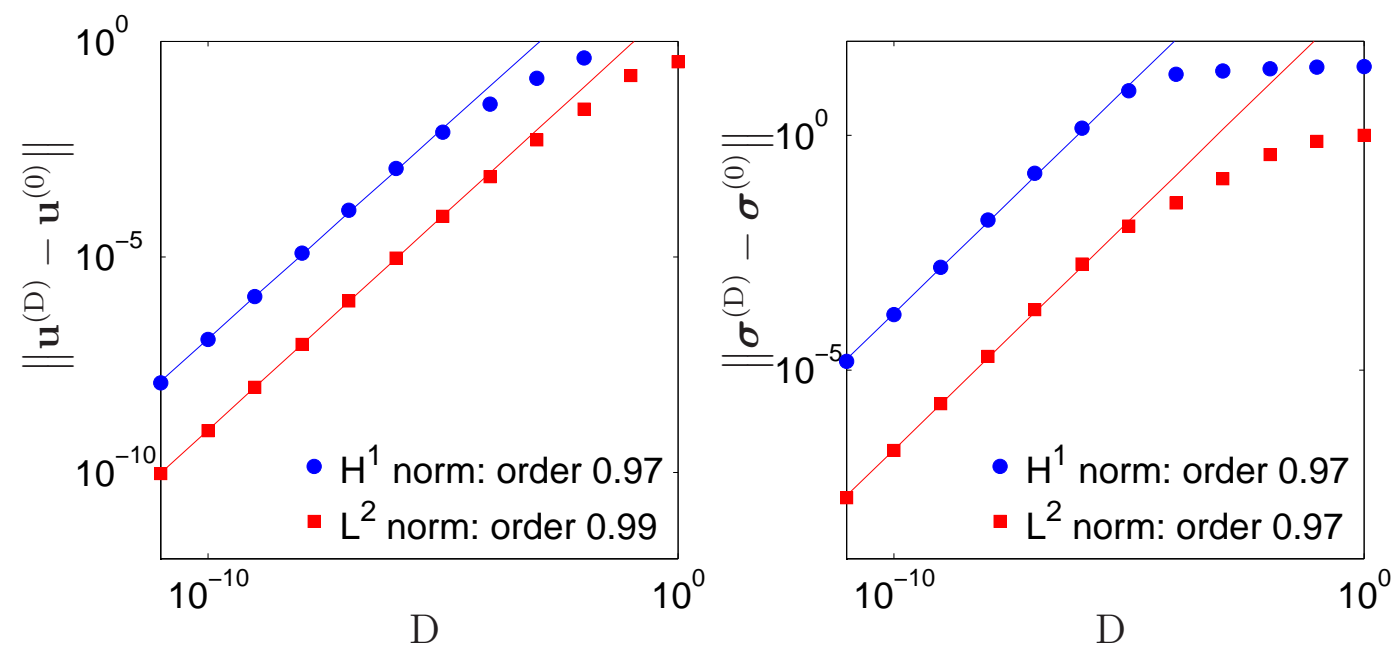

Figure 11: Lid-driven cavity in $2 \mathrm{D}$ with $\mathrm{Re}=5.0$, We $=0.5, r=0.5, a=0$ : influence of the diffusive term on the error on the velocity (1.) and the elastic constraint (r.). 

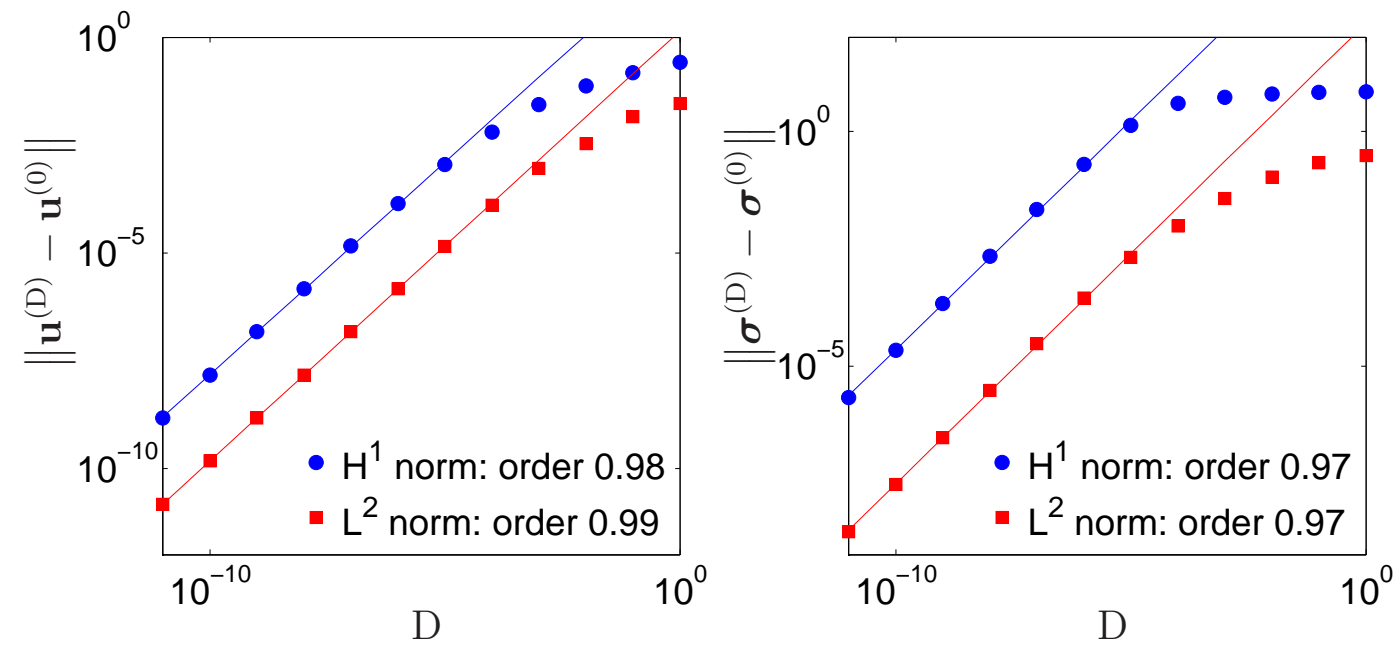

Figure 12: Lid-driven cavity in $2 \mathrm{D}$ with $\mathrm{Re}=5.0, \mathrm{We}=1.0, r=0.2, a=0$ : influence of the diffusive term on the error on the velocity (1.) and the elastic constraint (r.).
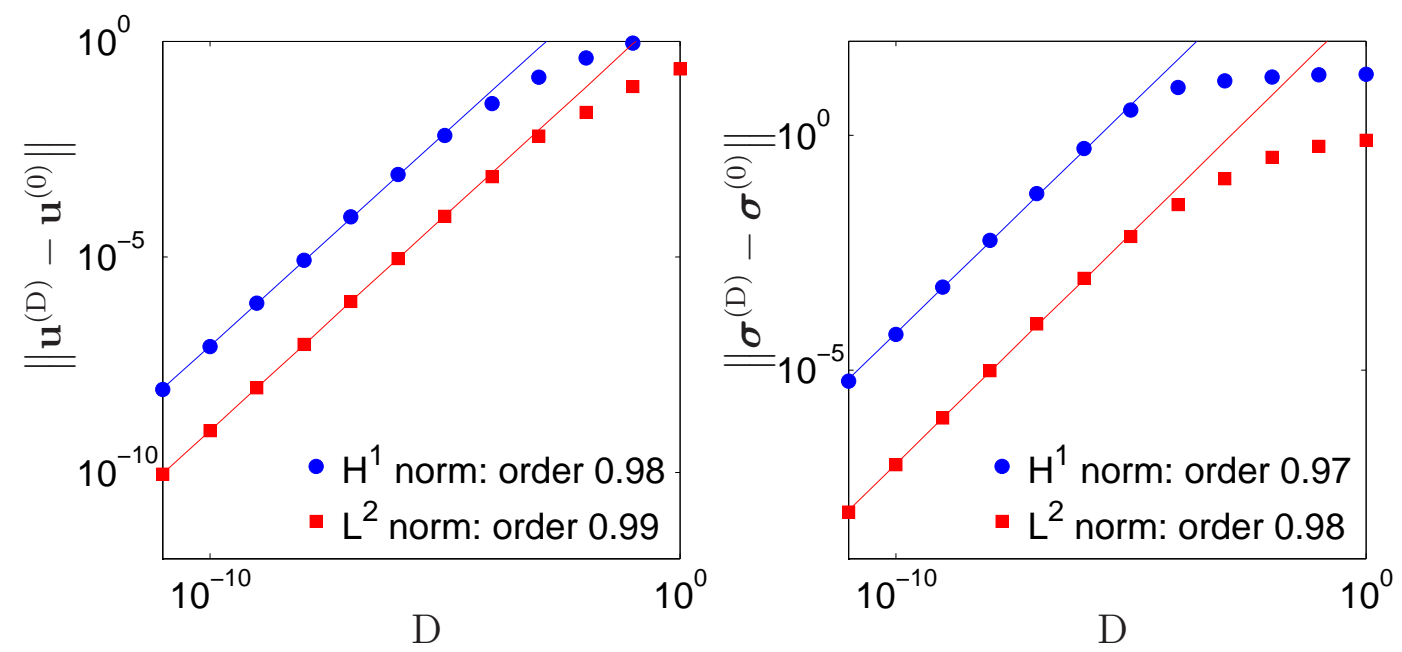

Figure 13: Lid-driven cavity in $2 \mathrm{D}$ with $\mathrm{Re}=5.0$, We $=1.0, r=0.5, a=0$ : influence of the diffusive term on the error on the velocity (1.) and the elastic constraint (r.). 

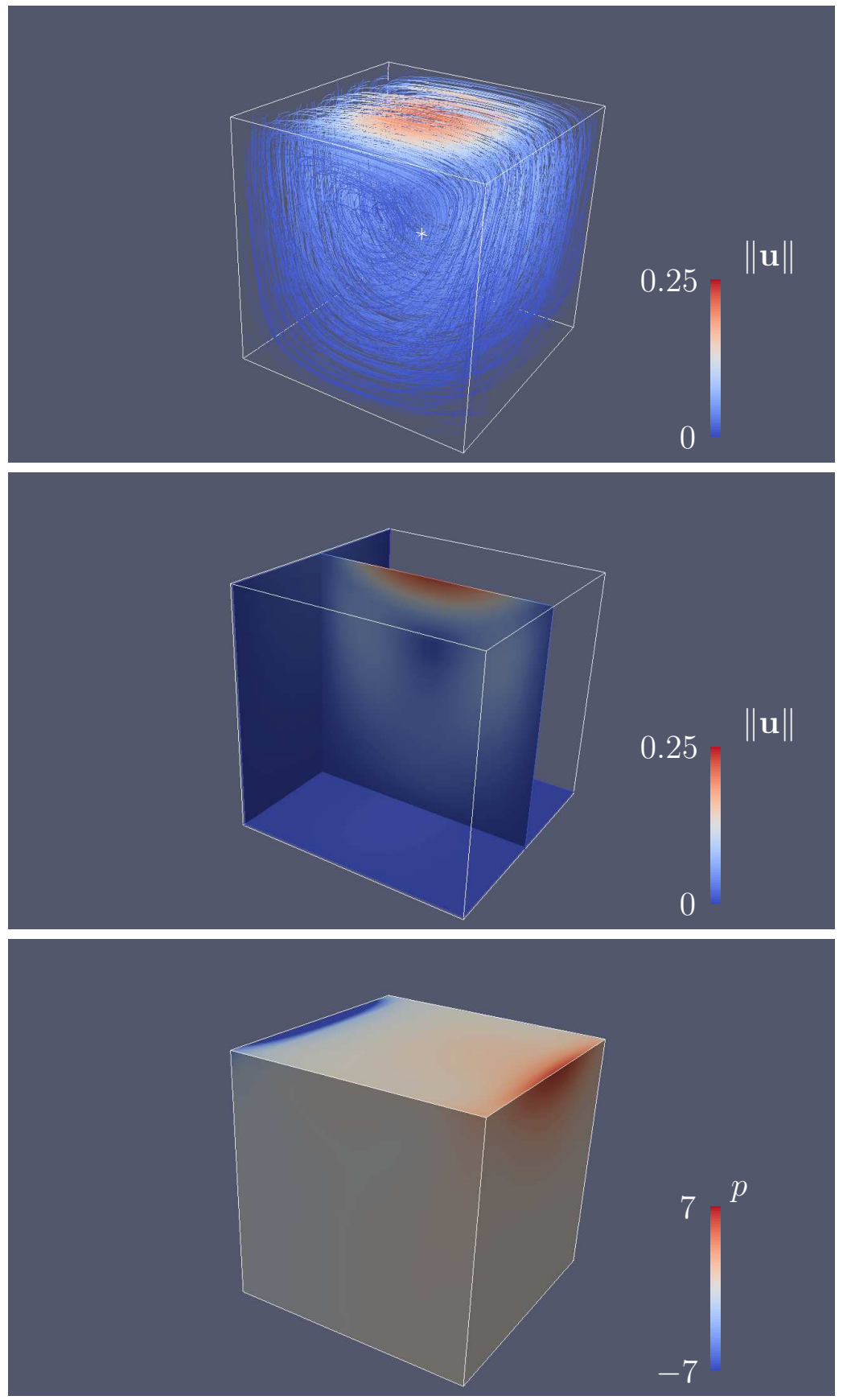

Figure 14: Solution of the stationary Oldroyd model for the 3D driven cavity problem with $\mathrm{Re}=4.0$, We $=1.0, r=0.5, a=0, \mathrm{D}=0.0$ : (t.) streamlines related to the velocity field; (m.) cutting planes sampling the 3D magnitude of the velocity field ; (b.) magnitude of the pressure field. 

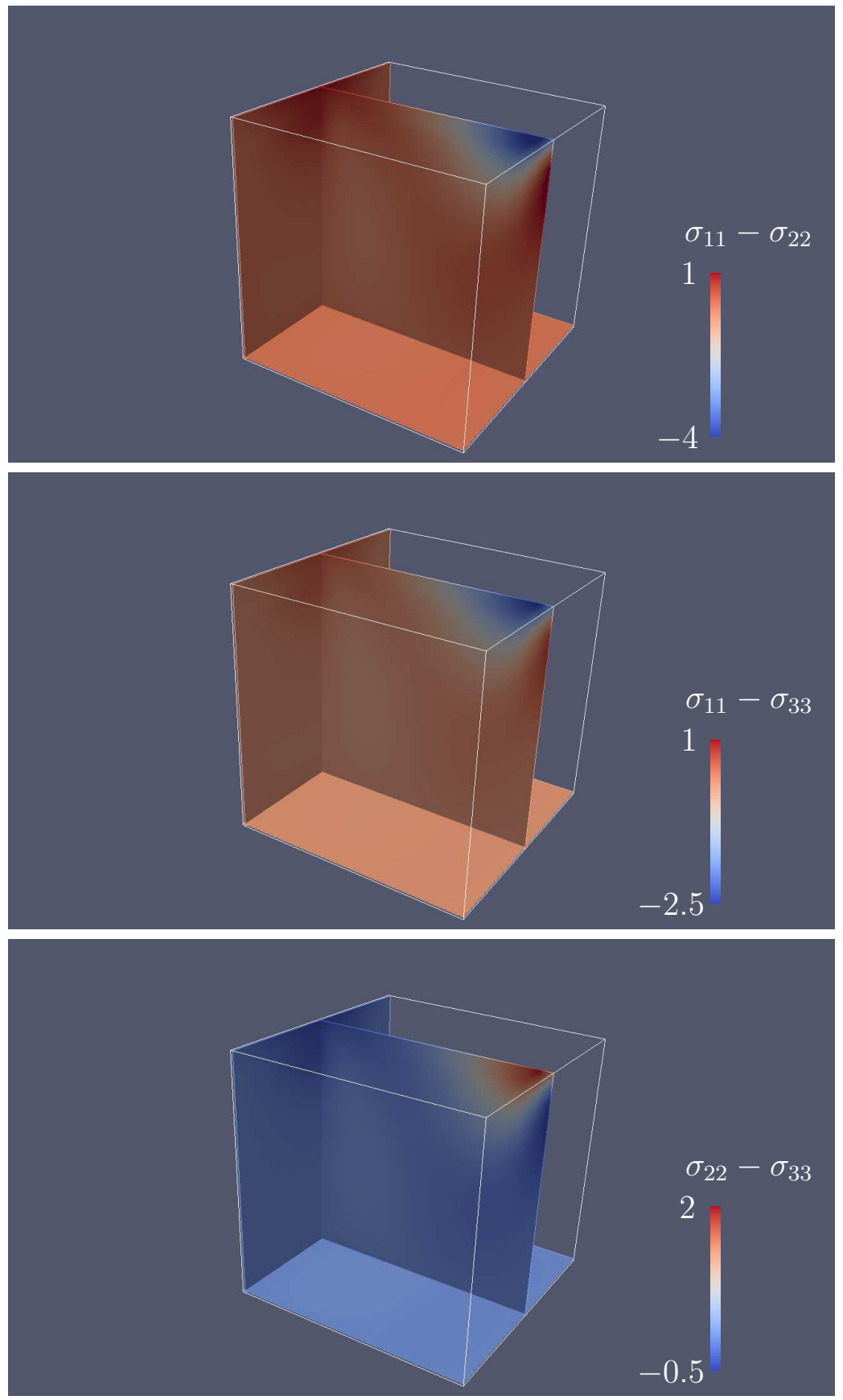

Figure 15: Solution of the stationary Oldroyd model for the 3D driven cavity problem with $\mathrm{Re}=4.0$, We $=1.0, r=0.5, a=0, \mathrm{D}=0.0$ : cutting planes sampling the elongational components of the elastic tensor (t.) $\sigma_{11}-\sigma_{22}$, (m.) $\sigma_{11}-\sigma_{33}$ and (b.) $\sigma_{22}-\sigma_{33}$. 

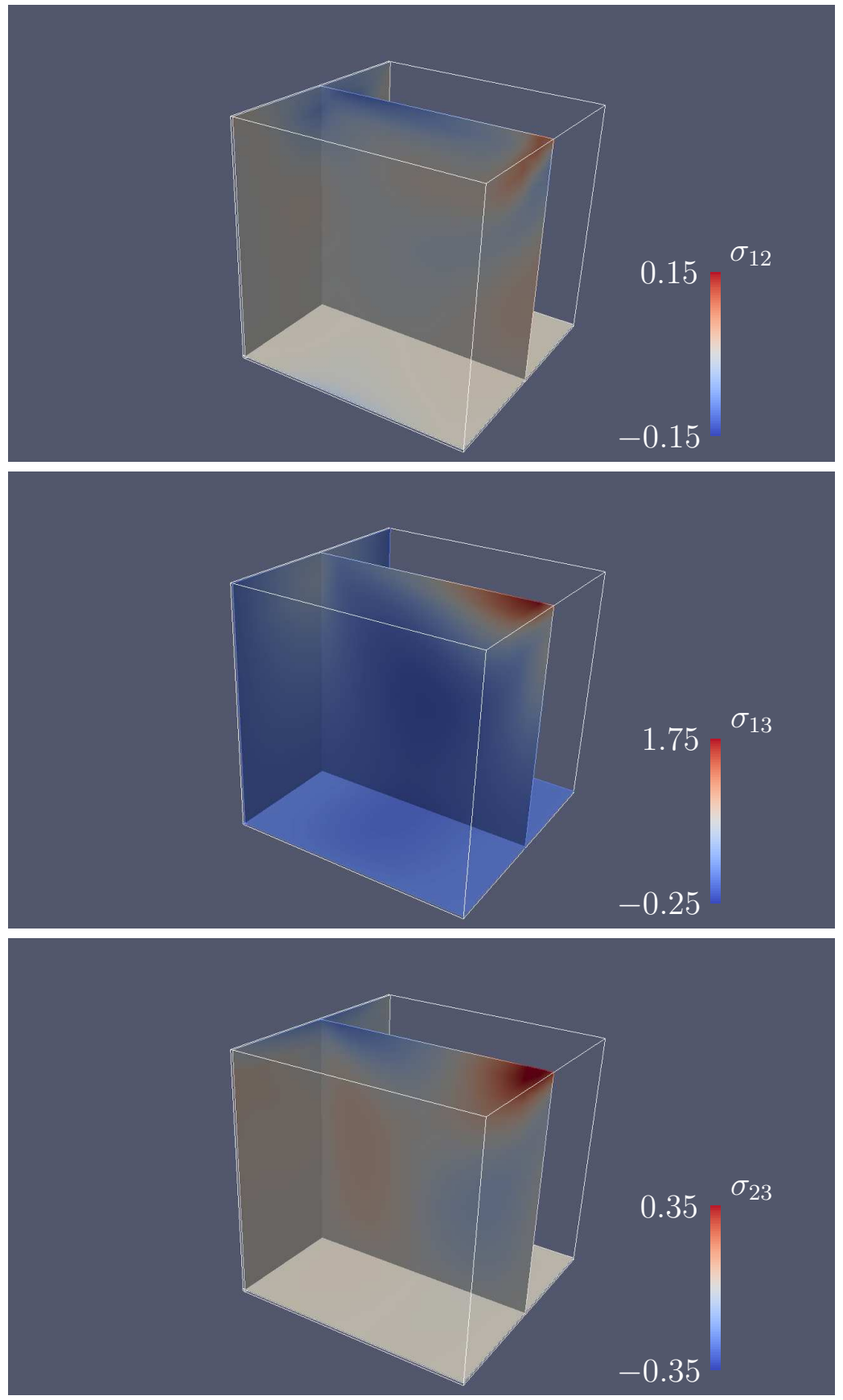

Figure 16: Solution of the stationary Oldroyd model for the 3D driven cavity problem with $\mathrm{Re}=4.0$, We $=1.0, r=0.5, a=0, \mathrm{D}=0.0$ : cutting planes sampling shear components of the elastic tensor (t.) $\sigma_{12}$, (m.) $\sigma_{13}$ and (b.) $\sigma_{23}$. 

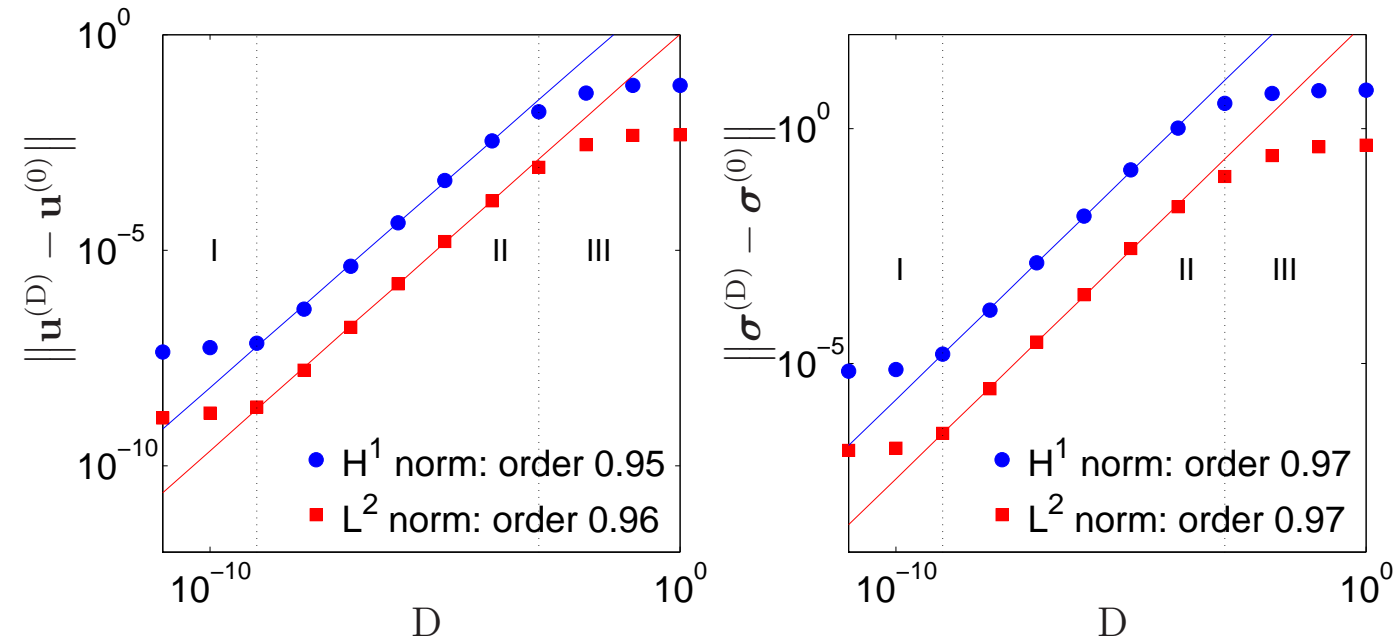

Figure 17: Lid-driven cavity in 3D: influence of the diffusive term on the error on the velocity (1.) and the elastic constraint (r.). Computations have been led with $\mathrm{We}=1.0, r=0.5, a=0$ in the non-inertial regime $\mathrm{Re}=0.0$.
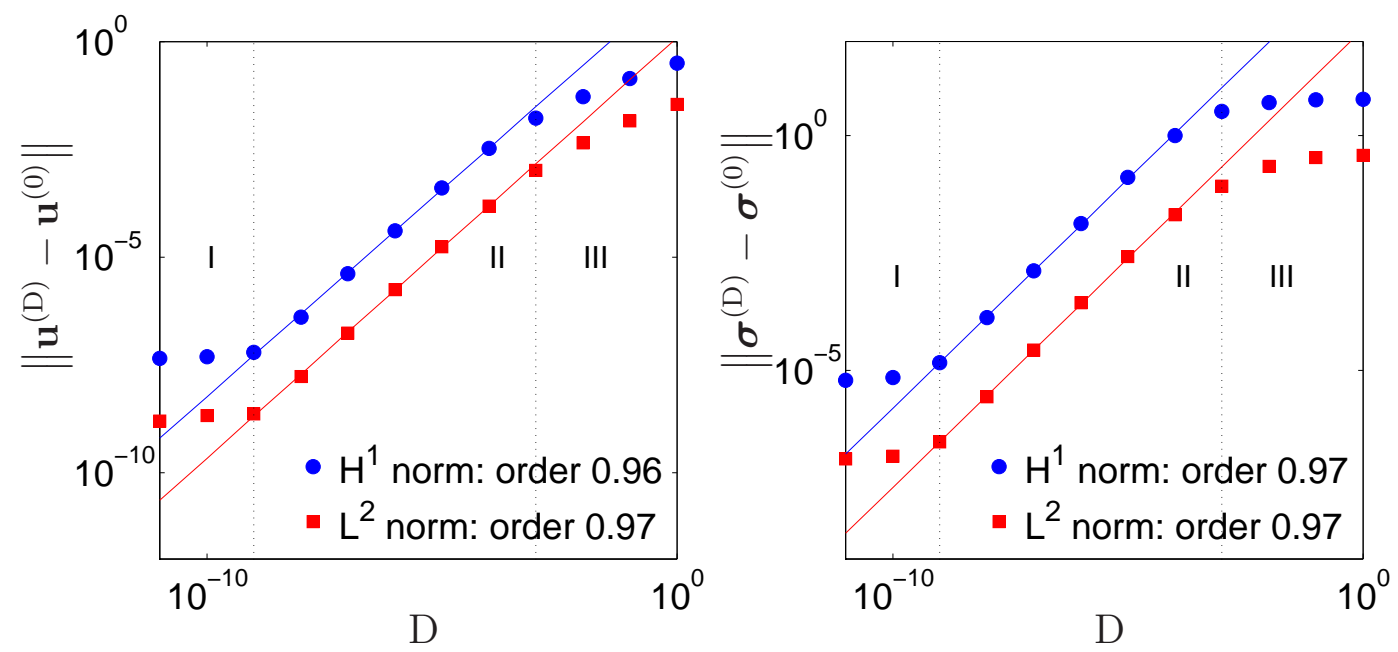

Figure 18: Lid-driven cavity in 3D: influence of the diffusive term on the error on the velocity (1.) and the elastic constraint (r.). Computations have been led with $\mathrm{We}=1.0, r=0.5, a=0$ in the inertial regime $\mathrm{Re}=4.0$. 